\title{
Article \\ Rapeseed Morpho-Physio-Biochemical Responses to Drought Stress Induced by PEG-6000
}

\author{
Maria Batool ${ }^{1}$, Ali Mahmoud El-Badri ${ }^{1,2}$, Zongkai Wang ${ }^{1}$, Ibrahim A. A. Mohamed 1,3 ${ }^{\mathbb{D}}$, Haiyun Yang ${ }^{1}$, \\ Xueying Ai ${ }^{1}$, Akram Salah ${ }^{1} \mathbb{D}$, Muhammad Umair Hassan ${ }^{4}$, Rokayya Sami ${ }^{5} \mathbb{D}$, Jie Kuai ${ }^{1}$, Bo Wang ${ }^{1, *(\mathbb{D})}$ \\ and Guangsheng Zhou ${ }^{1}$
}

1 MOA Key Laboratory of Crop Ecophysiology and Farming System in the Middle Reaches of the Yangtze River, College of Plant Science \& Technology, Huazhong Agricultural University, Wuhan 430070, China; maria.batool@webmail.hzau.edu.cn (M.B.); alyelbadry@webmail.hzau.edu.cn (A.M.E.-B.); wangzongkai@webmail.hzau.edu.cn (Z.W.); iaa04@fayoum.edu.eg (I.A.A.M.); comeonyhy@163.com (H.Y.); aixueying1994@163.com (X.A.); akramsaleh2002@mail.hzau.edu.cn (A.S.); kuaijie@mail.hzau.edu.cn (J.K.); zhougs@mail.hzau.edu.cn (G.Z.)

2 Field Crops Research Institute, Agricultural Research Center (ARC), Giza 12619, Egypt

3 Botany Department, Faculty of Agriculture, Fayoum University, Fayoum 63514, Egypt

4 Research Centre on Ecological Sciences, Jiangxi Agricultural University, Nanchang 330045, China; muhassanuaf@gmail.com

5 Department of Food Science and Nutrition, College of Sciences, Taif University, P.O. Box 11099, Taif 21944, Saudi Arabia; rokayya.d@tu.edu.sa

* Correspondence: wangbo@mail.hzau.edu.cn

\section{check for} updates

Citation: Batool, M.; El-Badri, A.M.; Wang, Z.; Mohamed, I.A.A.; Yang, H.; Ai, X.; Salah, A.; Hassan, M.U.; Sami, R.; Kuai, J.; et al. Rapeseed Morpho-Physio-Biochemical Responses to Drought Stress Induced by PEG-6000. Agronomy 2022, 12, 579. https://doi.org/10.3390/ agronomy12030579

Academic Editor: María del Pilar Cordovilla

Received: 14 January 2022

Accepted: 15 February 2022

Published: 26 February 2022

Publisher's Note: MDPI stays neutral with regard to jurisdictional claims in published maps and institutional affiliations.

Copyright: (C) 2022 by the authors. Licensee MDPI, Basel, Switzerland. This article is an open access article distributed under the terms and conditions of the Creative Commons Attribution (CC BY) license (https:// creativecommons.org/licenses/by/ $4.0 /)$.

\begin{abstract}
Rapeseed is a valuable oil crop due to its high nutritious value and ample oil content. The current study provides a comparative analysis of 24 cultivars to better understand the performance and predict the adaptative mechanisms of drought-tolerant and drought-sensitive cultivars based on germination and morphophysiological traits during the early seedling stage using PEG-6000 simulated drought conditions. JYZ 158 and FY 520 (tolerant cultivars) and YG 2009 and NZ 1838 (sensitive cultivars) were selected to further explore the role of osmolytes and enzymatic activity in improving drought tolerance. This investigation illustrated that drought stress negatively influenced all studied cultivars; however, the degree of influence was different for each cultivar, suggesting their different potential for drought tolerance. Moreover, enzymatic and osmoregulatory mechanisms were highly efficient in tolerant cultivars compared to sensitive cultivars. Additionally, tolerant cultivars showed higher chlorophyll and lower malondialdehyde (MDA) contents versus sensitive cultivars under drought stress conditions. Higher drought tolerance coincided with higher enzymatic activity and osmolyte content. This work showed that JYZ 158 and FY 520 cultivars had higher drought tolerance, and might be a significant germplasm resource for breeding programs developing drought-tolerant rapeseed.
\end{abstract}

Keywords: rapeseed; drought; early seedling stage; osmolytes; antioxidant enzymes

\section{Introduction}

Rapeseed (Brassica napus L.) is a valuable and economically important oilseed crop globally, occupying a large cultivation area in China with more than 7 million hectares [1]. It is one of the most important crops for global oil production and is a multipurpose edible crop [2]. Rapeseed meal is a valuable animal feed in the feed industry. Moreover, it has nutritional importance due to its ideal amino acid content, higher fiber content, and contents of essential vitamins and minerals [3]. It is susceptible to drought stress, which is detrimental at each developmental phase of the plant life cycle [4].

Water deficit is one of the crucial limiting factors which reduces crop growth and productivity [5]. China is hit badly by drought events, which directly affect the economy, causing losses higher than 4.78 billion euro (according to the 2018 price level). An area 
of more than 200 thousand $\mathrm{km}^{2}$ was affected between 1984 and 2018 (China Meteorological Administration, 2019). Drought stress is a critical abiotic factor that damages plants, increases oxidative stress and reduces plant height [6]. Furthermore, it negatively affects morpho-physiochemical processes and metabolic responses [7]. Drought-stressed rapeseed seedlings show a decrease in germination percentage, poor growth and vigor index with lower biomass accumulation [8], along with severe oxidative damage and impaired antioxidant defense systems [9].

Seed germination is an essential biological process in the growth cycle of plants [10]. In semi-arid areas, successful crop production is mainly dependent on optimum seed germination and early seedling growth that is closely linked with the capacity of seeds to sprout under drought stress [11]. Several physiochemical processes associated with moisture availability, stored material mobilization, hormonal activities and protein structure are affected under drought conditions, affecting seedling survival and growth [12]. It is widely documented that initial drought stress restricts seed germination leading to poor stand establishment of seedlings during development, hence impairing the crop growth $[13,14]$.

Drought conditions have inhibitory effects on rapeseed growth, impairing photosynthetic processes, leaf water content and subsequent developmental processes [15]. Additionally, the decline in photosynthetic pigments can be associated with a lower water supply, which reduces leaf water content [16]. Water deficiency causes chloroplasts to become oval to round in shape and move toward the center of the cell, indicating that drought impairs structural integrity [17]. Malondialdehyde (MDA) is the product of peroxidation of lipids in the membrane and an indicator of various stresses [18].

The generation of reactive oxygen species (ROS) is a responsive action taken by plants $[19,20]$. The equilibrium between synthesis and degeneration of ROS is not maintained under drought stress; hence, ROS (free radicals) accumulate in the cells, leading to cell membrane dysfunction [21]. Drought stress-induced lipid peroxidation enhances ROS production and breaks down unsaturated fatty acids, ultimately causing structural degradation of the seed and arresting seed germination [22]. Malondialdehyde (MDA) is the product of lipid peroxidation, and proline is one of the antioxidants which maintain cell turgor via osmotic adjustment and regulate redox metabolic processes to scavenge ROS [11].

Plants have a complicated enzymatic defensive mechanism against oxidative stress to suppress ROS overproduction that is correlated with tolerance against unfavorable conditions [23]. Osmotic substances play protective roles for membrane and assist the plant in water intake for maintaining physiological functioning [24]. Moreover, antioxidant enzymes, including superoxide dismutase (SOD) and peroxidase (POD), defend the cell membrane from oxidative damage by removing excessive ROS from cells under stress conditions [22]. Notably, catalase (CAT) and ascorbate peroxidase (APX) alleviate the damaging effects of stress [25]. The accumulation of osmolytes, such as proline, soluble sugars and protein, upon drought stress is linked to stress tolerance [18,26]. Moreover, total soluble sugar (TSS) and total soluble protein (TSP) are two important osmo-protectants that can help the plant withstand unfavorable environments [19].

It is important to identify drought-tolerant germplasm before developing a drought tolerance breeding program. Therefore, the current study aimed to increase understanding of the influence of drought stress on morphophysiological attributes of rapeseed by measuring key factors such as seedling growth, photosynthetic pigments, osmolytes accumulation, lipid peroxidation and enzymatic antioxidants. Diversity in the ability of the most common rapeseed cultivars to withstand drought stress during seed germination and the early seedling stage was examined. Our results can be used for further analysis and subsequent research. 


\section{Material and Methods}

\subsection{Plant Materials and Growth Conditions}

A panel comprised of 24 rapeseed cultivars with different genetic backgrounds was selected based on agronomic performance, economic importance and cultivated area to study the deleterious effects of drought stress during the early seedling stage (Table S1). The experiment was carried out in bifactorial design using three replications with four biological replications. The first factor contained 24 cultivars, and the second factor involved drought stress using polyethylene glycol 6000 (PEG-6000). Polyethylene glycol 6000 is a high molecular weight compound that is unable to pass through the cell wall; therefore, it can regulate water potential in the cells by outward water flow from plant tissues into a concentrated solution [27].

\subsection{Germination Trails}

A pilot study was conducted to select the concentration of PEG-6000 that should be used for inducing drought levels in the screening of cultivars. Three cultivars, randomly selected, were subjected to different levels of drought $(0,5,10,15,20$ and 25\% PEG-6000) for seven days. The results were noted for final germination percentage (FG\%) and it was found that PEG-6000 with 5\% concentration was similar to $0 \%$, where the FG\% was $99.44,99.44$ and 93.88\% (normal conditions) and 99.44, 98.33 and 95.00\% (5\% PEG-6000) in YYZ 3, XZY 518 and GZ 1, respectively, indicating that a $5 \%$ concentration is too low. A $20 \%$ concentration showed significantly reduced FG\% (85.55, 88.88 and 80.55\%) in YYZ 3, XZY 518 and GZ 1, respectively, and inhibited seedling growth. The severe drought effect caused stunted growth and could not use to measure required plant attributes. By comparison, 25\% PEG6000 showed highly significantly lower FG\% (8.888, 20.00 and 24.44\%) in YYZ 3, XZY 518 and GZ 1, respectively, suggesting severe stress without growth (Table S3). The maximum visible response was noted at 15\%, and a slight difference noted at 10\% PEG-6000, which were used for further study.

Mature seeds of 24 cultivars were carefully selected and hand-picked based on uniform size, surface sterilized using 70\% ethanol ( $5 \mathrm{~min}$ ), rinsed ( 5 times) with distilled water, and dried using blot paper until constant weight. Sixty uniform and healthy seeds were sown in polyethylene boxes $(12 \times 12 \times 6 \mathrm{~cm})$ with three-layered sterilized filter paper with $15 \mathrm{~mL}$ of a solution of 0,10 or $15 \%$ PEG-6000 in each germination box. The experiment was carried out for seven days in a growth chamber (day/night temperature at $25 / 20{ }^{\circ} \mathrm{C}$ ) with $12 \mathrm{~h}$ light (13,000 lx) and 12 h dark, at Huazhong Agricultural University, Wuhan, Hubei, China.

\subsection{Assessment of Morphological Traits}

Seeds with a minimum radicle extrusion of $2 \mathrm{~mm}$ were considered germinated and were counted daily in each box for seven days. Final germination percentage (FG\%), germination rate (GR), vigor index I (VI (I)) and vigor index II (VI (II)) were measured at the seventh day of the early growth stage. A description is given in (Table S2), according to the equation provided by [28]. Seedlings were harvested on the seventh day, and 10 seedlings with a uniform appearance from each replication were used to measure root and shoot length. Shoot and root fresh weight were calculated from the same seedlings, then dry weight was measured after the samples were dried at $80^{\circ} \mathrm{C}$ to constant weight.

\subsection{Determination of Photosynthetic Pigments}

After 7-days of treatment, chlorophyll (chl) and carotenoid contents $\left(\mu \mathrm{g} \mathrm{g}^{-1} \mathrm{FW}\right)$ in fresh leaves were determined. First, $0.1 \mathrm{~g}$ FW was mixed with $80 \%$ acetone in test tubes, kept overnight, and then centrifuged. Afterwards, absorbance was noted using an ultraviolet spectrophotometer (UV-2100, UNIC, Shanghai, China) at 646, 663 and $480 \mathrm{~nm}$ [10].

\subsection{Determination of Relative Water Content (RWC)}

After sampling, small leaves from whole plants were weighed, maintained in distilled water overnight, then dried with blotted paper and the saturated leaves weighed (turgor 
weight). The weighed samples were dried for $48 \mathrm{~h}$ at $80^{\circ} \mathrm{C}$, and the dry weight was noted. Leaf relative water content was calculated using the equation according to [29].

$$
\mathrm{RWC}=\frac{(\text { Fresh weight }- \text { dry weight })}{(\text { Turgor weight }- \text { dry weight })} \times 100
$$

\subsection{Determination of Total Soluble Sugar, Total Soluble Protein, Proline, and MDA Contents}

Total soluble sugar was estimated in samples using the anthrone sulfuric acid method. Briefly, $0.1 \mathrm{~g}$ fresh weight of sample was mixed thoroughly with $10 \mathrm{~mL}$ water. Afterwards, the mixture was boiled for $30 \mathrm{~min}$ at $100^{\circ} \mathrm{C}$ followed by centrifugation. The supernatant was collected and mixed in sulfuric acid-anthrone reagent, then boiled for $10 \mathrm{~min}$ at $95-100{ }^{\circ} \mathrm{C}$ in a water bath and cooling. The absorption value was read on a spectrophotometer at $620 \mathrm{~nm}$ following the method of [30]. The Coomassie brilliant blue (CBB) method was used to estimate total soluble protein in the fresh sample, the absorbance value at $595 \mathrm{~nm}$ being read on a spectrophotometer following the method of [31].

Proline content was measured using the method described by [32]. Fresh shoots $(0.1 \mathrm{~g})$ were mixed with $3 \%$ aqueous sulfosalicylic acid and the homogenate was centrifuged. Then supernatants were mixed with glacial acetic acid and ninhydrin reagent and shaken thoroughly, then placed in a water bath for $30 \mathrm{~min}$ followed by cooling. The mixture was centrifuged at 10,000 rpm for $5 \mathrm{~min}$, extracted with $4 \mathrm{~mL}$ toluene followed by vortex mixing, and the absorption value was noted using a UV-spectrophotometer at $520 \mathrm{~nm}$. Proline content was measured using a standard curve [32].

Malondialdehyde (MDA) content measures lipid peroxidation, assessed by the Heath and Packer method [33]. Fresh shoot sample ( $0.5 \mathrm{~g})$ was homogenized with $5 \mathrm{~mL}$ of $0.1 \%$ $(w / v)$ trichloroacetic acid (TCA) and centrifuged for $20 \mathrm{~min}$. Supernatants were collected, and $4 \mathrm{~mL}$ of $0.5 \%$ thiobarbituric acid (TBA) in $20 \%$ TCA was added. Then, the reaction solution was subjected to heating for $30 \mathrm{~min}$ at $95{ }^{\circ} \mathrm{C}$ followed by cooling, centrifugation for 15 min and supernatants were collected carefully. The MDA content was calculated using a UV-spectrophotometer at 450, 532 and $600 \mathrm{~nm}$.

\subsection{Measurement of Antioxidant Enzyme Activities}

The activities of antioxidant enzymes were assessed by homogenizing $0.1 \mathrm{~g}$ of crushed frozen samples with potassium phosphate buffer (PPB) ( $\mathrm{pH} 7.8)$. The homogenate was centrifuged at $12,000 \mathrm{rpm}$ for $20 \mathrm{~min}$ at $4{ }^{\circ} \mathrm{C}$ to collect the supernatant. SOD, CAT, POD and APX activities were determined in the supernatant using a spectrophotometer with respective wavelengths according to the manufacturer's instructions, respectively, following the methods of [10].

Superoxide dismutase (SOD; EC 1.15.1.1) activity was assessed by inhibiting photochemical reduction by nitro blue tetrazolium (NBT). The reaction mixture contained $50 \mathrm{mM}$ PPB (pH 7.8), $13 \mathrm{mM}$ methionine, $75 \mathrm{mM}$ NBT, $2 \mathrm{mM}$ riboflavin, $0.1 \mathrm{mM}$ EDTA and $0.1 \mathrm{~mL}$ of enzyme extract in a $3 \mathrm{~mL}$ volume. One unit of SOD activity was measured as the amount of enzyme required to cause $50 \%$ inhibition of NBT reduction and was measured spectrophotometrically at $560 \mathrm{~nm}$.

To assay peroxidase (POD; EC 1.11.1.7) activity, $0.1 \mathrm{~mL}$ enzyme extract was mixed with $50 \mathrm{mM}$ PPB (pH 7.0), 1\% (m/v) guaiacol, and $0.4 \%(v / v) \mathrm{H}_{2} \mathrm{O}_{2}$. The absorbance was measured at a $470 \mathrm{~nm}$.

The assay for ascorbate peroxidase (APX; EC 1.11.1.11) was conducted using a reaction mixture (3 mL) containing $100 \mathrm{mM}$ phosphate ( $\mathrm{pH} 7), 0.1 \mathrm{mM}$ EDTA-Na2, $0.3 \mathrm{mM}$ ascorbic acid, $0.06 \mathrm{mM} \mathrm{H}_{2} \mathrm{O}_{2}$, and $0.1 \mathrm{~mL}$ enzyme extract. The change in absorption was quantified at $290 \mathrm{~nm}$ for $30 \mathrm{~s}$ after adding $\mathrm{H}_{2} \mathrm{O}_{2}$.

The method to measure catalase (CAT; EC 1.11.1.6) activity used $\mathrm{H}_{2} \mathrm{O}_{2}$ (extinction co-efficient $\left.39.4 \mathrm{mM}^{-1} \mathrm{~cm}^{-1}\right), 3 \mathrm{~mL}$ reaction mixture containing $50 \mathrm{mM}$ PPB ( $\left.\mathrm{pH} 7.0\right)$, $2 \mathrm{mM}$ EDTA-Na, $10 \mathrm{mM} \mathrm{H}_{2} \mathrm{O}_{2}$, and $0.1 \mathrm{~mL}$ enzyme extract, the spectrophotometric assay recorded at $240 \mathrm{~nm}$. 


\subsection{Microstructural Analysis}

Fresh leaf samples were cleaned with distilled water and cut into uniform slices, fixed with $4 \%$ glutaraldehyde and $0.2 \mathrm{M}$ sodium phosphate buffer $(\mathrm{pH} 6.8)$ and then distilled with $0.1 \mathrm{M}$ sodium phosphate buffer ( $\mathrm{pH}$ 6.8). Phosphate buffer $(0.2 \mathrm{M}, \mathrm{pH} 6.8)$ was used to fix the sample. Afterwards, dehydration was done in a gradient ethanol series. Slices were examined using a transmission electron microscope after staining with lead citrate and $2 \%$ uranyl acetate [34].

\subsection{Statistical Analysis}

The experiment was carried out as a bifactorial design, and measurements were made with three replications. Statistical analysis for germination and growth-related traits was conducted using Statistix 8.1 software with linear models. Significant differences (LSD) were calculated to examine differences at $p<0.05$. Differences among treatments were determined using ANOVA. Graphical presentation was carried out using GraphPad prism (V: 5.0.1) and RStudio software.

\section{Results}

\subsection{Variation in Seed Germination Traits under Drought Stress}

The impact of drought stress on various 24 cultivars of rapeseed using different concentrations of PEG-6000 (0, 10 and 15\%) was studied. The mean values of FG\%, GR, VI (I) and VI (II) were measured to estimate the negative effect of drought stress on seed germination. Results showed that the mean values of all measured traits were significantly reduced at the higher level of drought stress (15\% PEG-6000) compared to the control (Table 1). Remarkably, few cultivars showed better performance under 10\% PEG-6000induced drought than under normal conditions. Box and whisker charts showed the variation in germination traits for all 24 rapeseed cultivars, measured under 0,10 and 15\% PEG-6000 treatments. Additionally, the box and whisker charts showed substantial variations of germination-related traits between treatments, especially at 15\% PEG 6000, indicated by the lower and upper limits of box plot for each trait (Figure 1). The mean of the measured traits showed a significant reduction at the higher level of drought stress (15\% PEG-6000). The mean values of FG\%, GR, V(I) and V(II) were 94.67\%, 33.00, 921.3 and 36.87 (normal conditions), 94.49\%, 27.02, 1056 and 26.49 (10\% PEG-6000), 83.07\%, 19.44, 706.0 and 15.65 (15\% PEG-6000), respectively (Table 1).

Table 1. Seed germination traits under different concentrations of PEG-6000-induced drought stress.

\begin{tabular}{|c|c|c|c|c|c|c|c|c|c|c|c|c|}
\hline \multirow{2}{*}{ Variety } & \multicolumn{3}{|c|}{ FG $\%$} & \multicolumn{3}{|c|}{ GR } & \multicolumn{3}{|c|}{ VI (I) } & \multicolumn{3}{|c|}{ VI (II) } \\
\hline & CK & $10 \%$ & $15 \%$ & CK & $10 \%$ & $15 \%$ & CK & $10 \%$ & $15 \%$ & CK & $10 \%$ & $15 \%$ \\
\hline CY 81 & $91.67 \mathrm{~b}-\mathrm{e}$ & 90.55 cde & $57.77^{j}$ & $20.76^{\mathrm{m}}$ & $20.76^{j}$ & $9.840^{1}$ & $976.7 \mathrm{de}$ & $1045 \mathrm{fgh}$ & $592.3 \mathrm{hi}$ & $33.33 \mathrm{~h}-\mathrm{k}$ & $27.38 \mathrm{~d}-\mathrm{h}$ & $10.33 \mathrm{k}$ \\
\hline YYZ 3 & $99.33^{\mathrm{a}}$ & $98.88^{\mathrm{ab}}$ & $99.44^{\mathrm{a}}$ & $43.42^{\mathrm{cd}}$ & $32.47^{\mathrm{c}}$ & $28.28 \mathrm{bc}$ & $1061^{\mathrm{cd}}$ & $1120^{c-f}$ & $878.7^{\mathrm{cd}}$ & $31.66^{\mathrm{klm}}$ & $20.55 \mathrm{j}$ & $16.54^{\mathrm{efg}}$ \\
\hline YY 28 & $97.77 \mathrm{abc}$ & 96.11 abc & $78.33^{\mathrm{h}}$ & $35.49^{\mathrm{f}}$ & 27.69 efg & $14.28 \mathrm{k}$ & $347.3^{j}$ & $520.3^{1}$ & $336.7^{\mathrm{j}}$ & $31.33 \mathrm{~lm}$ & $24.68 \mathrm{hi}$ & $13.67 \mathrm{hij}$ \\
\hline CY 36 & $99.33^{\mathrm{a}}$ & $98.88 \mathrm{ab}$ & 89.44 def & $30.95 \mathrm{hi}$ & $28.16 \mathrm{ef}$ & 23.44 ef & $804.3 \mathrm{gh}$ & $1102^{\mathrm{d}-\mathrm{g}}$ & $820.4 \mathrm{de}$ & $32.66^{\mathrm{jkl}}$ & $27.32 \mathrm{e}-\mathrm{h}$ & $18.33^{b}$ \\
\hline JYZ 158 & $96.67^{\mathrm{a}-\mathrm{d}}$ & $98.33 \mathrm{ab}$ & $97.77 \mathrm{ab}$ & $51.33^{\mathrm{a}}$ & $39.24^{\mathrm{a}}$ & $30.82 \mathrm{ab}$ & $1243^{\mathrm{ab}}$ & $1361^{\mathrm{a}}$ & $1041^{\mathrm{a}}$ & $45.66 \mathrm{ab}$ & $34.67^{\mathrm{a}}$ & $22.33^{\mathrm{a}}$ \\
\hline ZY 50 & $98.67^{\mathrm{a}}$ & $99.44 \mathrm{ab}$ & $96.66^{a b c}$ & 28.29 ij & 27.64 efg & $22.53 \mathrm{fg}$ & $942.1 \mathrm{~d}-\mathrm{g}$ & $1245 \mathrm{bc}$ & 813.3 de & $32.66^{\mathrm{jkl}}$ & $26.66^{\mathrm{e}-\mathrm{h}}$ & $14.80^{\mathrm{f}-\mathrm{i}}$ \\
\hline QY 33 & $96.67^{\mathrm{a}-\mathrm{d}}$ & $100.0^{\mathrm{a}}$ & $85.56{ }^{\mathrm{efg}}$ & $42.54 \mathrm{~cd}$ & $33.48^{\mathrm{c}}$ & $20.36^{\mathrm{ghi}}$ & $1133^{c}$ & $1388^{\mathrm{a}}$ & $881.7^{\text {efg }}$ & $37.66^{\mathrm{d}-\mathrm{h}}$ & $23.68^{1}$ & $14.67^{\mathrm{f}-\mathrm{i}}$ \\
\hline ZY 51 & $96.67^{a-d}$ & $96.11 \mathrm{a}-\mathrm{d}$ & $67.67^{\mathrm{i}}$ & $25.22 \mathrm{k}$ & $24.72 \mathrm{hi}$ & $13.99 \mathrm{k}$ & $873 . \mathrm{e}^{\mathrm{efg}}$ & $976.5 \mathrm{hi}$ & $509.6^{\mathrm{i}}$ & $40.33 \mathrm{~cd}$ & $26.89 \mathrm{e}-\mathrm{h}$ & $13.67^{\mathrm{ij}}$ \\
\hline XZY 518 & $99.33^{\mathrm{a}}$ & $99.44 \mathrm{ab}$ & 91.30 b-e & $41.25 \mathrm{~d}$ & $31.56^{\mathrm{cd}}$ & $22.36 \mathrm{fg}$ & $973.7 \mathrm{def}$ & $1087 \mathrm{gh}$ & $712.3^{\mathrm{fg}}$ & $37.66^{\mathrm{d}-\mathrm{i}}$ & $27.01 \mathrm{f}-\mathrm{h}$ & $18.46^{b c}$ \\
\hline GHY 8 & $89.33 \mathrm{e}$ & $89.44 \mathrm{def}$ & $88.88 \mathrm{def}$ & $24.94 \mathrm{kl}$ & $24.95 \mathrm{hi}$ & $20.83 \mathrm{gh}$ & $746.3^{\mathrm{h}}$ & $788.1^{\mathrm{j}}$ & $687.5 \mathrm{gh}$ & $35.86^{\mathrm{i}-1}$ & $23.60^{1}$ & $13.66^{\mathrm{ij}}$ \\
\hline ZYZ 108 & 91.11 cde & $88.33 \mathrm{ef}$ & $79.33 \mathrm{hg}$ & $33.28 \mathrm{fg}$ & $24.79 \mathrm{hi}$ & $17.63^{\mathrm{j}}$ & $595.1^{\mathrm{i}}$ & $976.6 \mathrm{hi}$ & $617.3^{\mathrm{h}}$ & $37.58^{\mathrm{e}-\mathrm{i}}$ & 29.38 cde & $15.79 \mathrm{e}-\mathrm{h}$ \\
\hline NZ 1838 & $90.00 \mathrm{de}$ & $83.88^{\mathrm{f}}$ & $55.67^{\mathrm{j}}$ & $16.93^{\mathrm{n}}$ & $15.26^{\mathrm{k}}$ & $7.251^{\mathrm{m}}$ & $839.1 \mathrm{fgh}$ & $921.1^{\mathrm{i}}$ & $259.3^{j}$ & $32.64^{\mathrm{jkl}}$ & $20.55^{\mathrm{j}}$ & $7.336^{1}$ \\
\hline XZY 553 & $98.67 \mathrm{ab}$ & $99.44 \mathrm{ab}$ & $96.67 \mathrm{abc}$ & $43.86^{\mathrm{c}}$ & $32.37^{\mathrm{c}}$ & $25.47 \mathrm{de}$ & $987.7 \mathrm{de}$ & $1070^{\mathrm{e}-\mathrm{h}}$ & $922.3 \mathrm{bc}$ & $41.66 \mathrm{bc}$ & $32.28 \mathrm{bc}$ & $18.51 \mathrm{bc}$ \\
\hline YY 9 & $99.33^{\mathrm{a}}$ & $96.66 \mathrm{abc}$ & $78.33^{\mathrm{h}}$ & $31.69 \mathrm{gh}$ & $22.32^{\mathrm{il}}$ & $14.99 \mathrm{k}$ & $810.7 \mathrm{gh}$ & $710.6^{\mathrm{jk}}$ & $352.7^{\mathrm{j}}$ & $38.66 \mathrm{~d}^{\mathrm{ef}}$ & $20.33^{j}$ & $12.67^{\mathrm{j}}$ \\
\hline HYZ 62 & $96.67^{a-d}$ & $97.77 \mathrm{ab}$ & 88.33 def & $41.83 \mathrm{~d}$ & $29.98 \mathrm{de}$ & 19.90 hij & 973.7 de & $985.7 \mathrm{ghi}$ & $607.3 \mathrm{hi}$ & $48.43^{\mathrm{a}}$ & $32.28 \mathrm{ab}$ & 17.33 cde \\
\hline QY3 & $95.67^{a-e}$ & $94.44^{\mathrm{a}-\mathrm{d}}$ & $82.67^{\mathrm{fgh}}$ & $38.62 \mathrm{e}$ & $28.58 \mathrm{ef}$ & $14.10^{\mathrm{k}}$ & $1127^{c}$ & $1298 \mathrm{ab}$ & $818.2 \mathrm{de}$ & $38.66 \mathrm{~d}^{\mathrm{ef}}$ & $26.67^{\mathrm{e}-\mathrm{h}}$ & $16.67 \mathrm{cde}$ \\
\hline QY 7 & $90.00 \mathrm{de}$ & 90.55 cde & 90.00 cde & $33.35 \mathrm{fg}$ & $25.61 \mathrm{gh}$ & $22.65^{\mathrm{fg}}$ & $822.7 \mathrm{gh}$ & $976.0 \mathrm{hi}$ & $883.3^{\mathrm{bcd}}$ & $28.66^{\mathrm{m}}$ & $20.66^{\mathrm{j}}$ & $16.33^{\text {cde }}$ \\
\hline ZS 11 & $96.11^{\mathrm{a}-\mathrm{e}}$ & $97.77^{b}$ & $93.67^{a-d}$ & $32.70 \mathrm{gh}$ & $26.60^{\mathrm{fgh}}$ & $26.67 \mathrm{~cd}$ & $956.3^{\mathrm{d}-\mathrm{g}}$ & $1072 \mathrm{fgh}$ & $1033^{\mathrm{a}}$ & $35.477^{g-j}$ & 29.33 def & 16.33 cde \\
\hline YG 2009 & $66.67^{\mathrm{f}}$ & $66.66 \mathrm{~g}$ & $28.67 \mathrm{k}$ & $9.352^{\circ}$ & $10.51^{1}$ & $4.661^{\mathrm{n}}$ & $540.3^{\mathrm{i}}$ & $606.4 \mathrm{kl}$ & $162.4^{\mathrm{k}}$ & $21.69^{\mathrm{n}}$ & $14.36^{\mathrm{k}}$ & $3.330 \mathrm{~m}$ \\
\hline HYZ 72 & $93.33^{\text {a }-\mathrm{e}}$ & $96.66^{\mathrm{abc}}$ & 88.33 def & $27.65^{j}$ & $25.31 \mathrm{gh}$ & $17.84 \mathrm{ij}$ & $1046^{\mathrm{cd}}$ & $1351 \mathrm{ab}$ & $787.7^{\mathrm{ef}}$ & $37.69 \mathrm{~d}-\mathrm{g}$ & $27.59 \mathrm{fgh}$ & $15.67^{\mathrm{f}-\mathrm{i}}$ \\
\hline QY 1 & $98.67 \mathrm{ab}$ & $96.11^{\mathrm{a}-\mathrm{d}}$ & 88.33 fgh & $31.31 \mathrm{gh}$ & 27.68 efg & $20.64 \mathrm{gh}$ & $1133^{c}$ & $1176^{\mathrm{cd}}$ & $972.3^{\mathrm{a}}$ & $36.39 \mathrm{e}-\mathrm{i}$ & 26.33 ghi & 17.33 cde \\
\hline TYZ 283 & $96.67^{\mathrm{a}-\mathrm{e}}$ & $97.77^{\mathrm{ab}}$ & 82.67 cde & $22.64^{\mathrm{Im}}$ & $20.90^{\mathrm{j}}$ & $13.92 \mathrm{k}$ & $1036^{\mathrm{cd}}$ & $1137^{\text {cde }}$ & $587.7 \mathrm{hi}$ & $35.68^{f-j}$ & $27.51^{d-g}$ & $14.51 \mathrm{ghi}$ \\
\hline GZ 1 & $93.67^{a-e}$ & $93.88^{b-e}$ & 90.00 cde & $38.35^{\mathrm{e}}$ & $31.55^{\mathrm{cd}}$ & $22.59 \mathrm{fg}$ & $1136 \mathrm{bc}$ & $1325 \mathrm{ab}$ & $974.3 \mathrm{ab}$ & 38.77 de & $29.30 \mathrm{~cd}$ & $17.68^{b c d}$ \\
\hline
\end{tabular}


Table 1. Cont.

\begin{tabular}{|c|c|c|c|c|c|c|c|c|c|c|c|c|}
\hline \multirow{2}{*}{ Variety } & \multicolumn{3}{|c|}{ FG\% } & \multicolumn{3}{|c|}{ GR } & \multicolumn{3}{|c|}{ VI (I) } & \multicolumn{3}{|c|}{ VI (II) } \\
\hline & CK & $10 \%$ & $15 \%$ & CK & $10 \%$ & $15 \%$ & CK & $10 \%$ & $15 \%$ & CK & $10 \%$ & $15 \%$ \\
\hline FY 520 & $98.88^{\mathrm{a}}$ & $100.0^{\mathrm{a}}$ & $98.30 \mathrm{ab}$ & $46.36^{\mathrm{b}}$ & $36.43 \mathrm{~b}$ & $32.28^{\mathrm{a}}$ & $1281^{a}$ & $1088^{\mathrm{d}-\mathrm{g}}$ & $896.7 \mathrm{bcd}$ & $47.44^{\mathrm{a}}$ & $32.67 \mathrm{ab}$ & $23.78^{a}$ \\
\hline Mean & 94.67 & 94.49 & 83.07 & 33.00 & 27.02 & 19.44 & 921.3 & 1056 & 706.01 & 36.87 & 26.49 & 15.65 \\
\hline
\end{tabular}

A

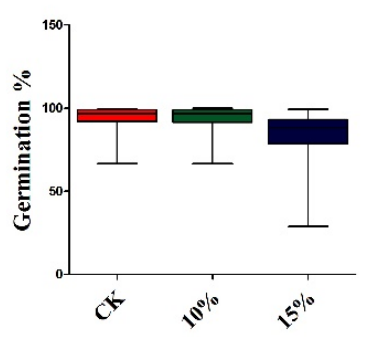

E

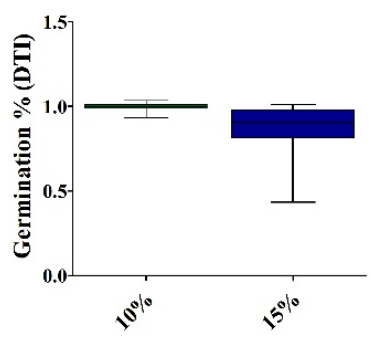

B

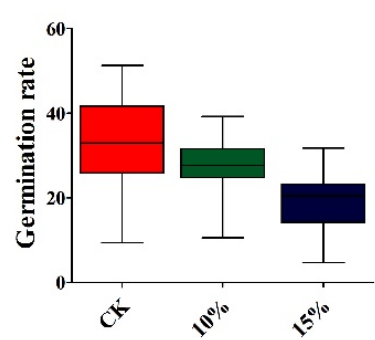

F

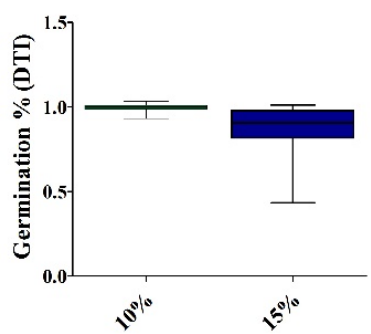

C

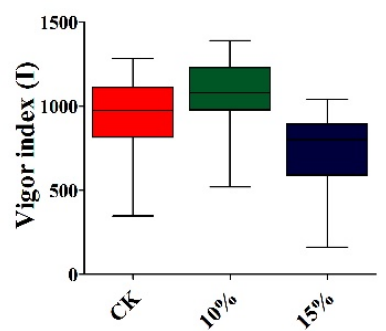

G

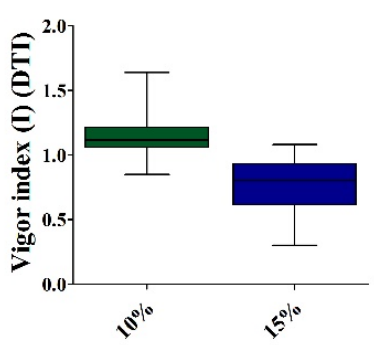

D

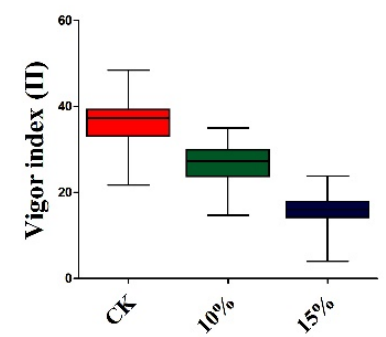

H

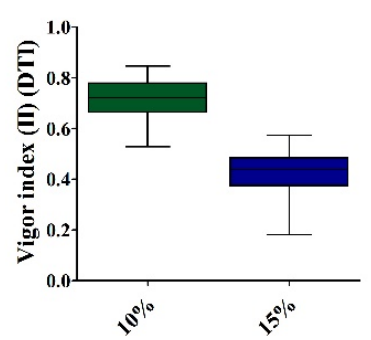

Figure 1. Box and whisker charts showing variation in germination-related traits: (A) final germination \%, (B) germination rate, (C) vigor index (I) and (D) vigor index (II), under CK, 10\%, and 15\% PEG-6000 treatments. Reduction in DTI values of (E) final germination \%, (F) germination rate, (G) vigor index (I), and (H) vigor index (II) under drought stress in rapeseed seedlings.

FG\% was greatly affected by the 15\% PEG-6000 concentration. YG 2009, NZ 1838 and CY 81 had the lowest values at $28.67,55.67$ and $57.77 \%$, respectively. Some cultivars had higher values, including YYZ 3 (99.44\%), JYZ 158 (97.77\%) and FY 520 (98.30\%) under 15\% PEG-6000. The germination rate (GR) was lowest in YG 2009 and NZ 1838, at 4.661 and 7.251, respectively, while few cultivars showed better performance of GR, including JYZ 158 and FY 520 with 30.82 and 32.28 values, respectively, under the 15\% PEG-6000 treatment (Table 1).

Vigor index decreased under the higher PEG-6000 concentrations (especially 15\% PEG-6000), while, cultivars with comparatively higher vigor index values showed better adaptability. JYZ 158 and FY 520 had the highest values of 1041 and 896.7 (VI (I)), 22.33, and 23.78 (VI (II)) under 15\% PEG-6000, respectively. On the other hand, few cultivars showed lower values of VI (I) and (II): YG 2009 (162.4 and 3.330) and NZ 1838 (259.3 and 7.336) under 15\% PEG-6000, respectively (Table 1).

\subsection{Variation in Seedling Growth Traits under Drought Stress}

The box and whisker charts revealed substantial variations of seedling growth traits between treatments, especially at 15\% PEG 6000, indicated by lower and upper limits of box plot for each trait (Figure 2). Under normal conditions, the mean values were recorded as $0.266,0.122,0.089,0.0084,2.235$ and 7.470 , while being $0.188,0.090,0.032,0.0094,1.672$ and 9.463 under 10\% PEG-6000 treatment, and 0.140, 0.045, 0.031, 0.0056, 1.337 and 6.968 under the 15\% PEG-6000 treatment for shoot fresh weight (ShFW), root fresh weight (RFW), 
shoot dry weight (ShDW), root dry weight (RDW), shoot length (ShL) and root length (RL), respectively (Table 2).

A

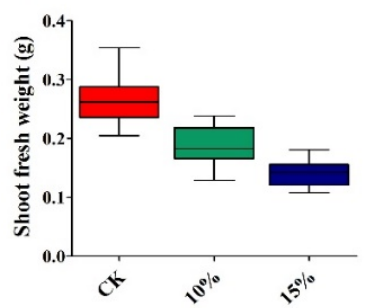

E
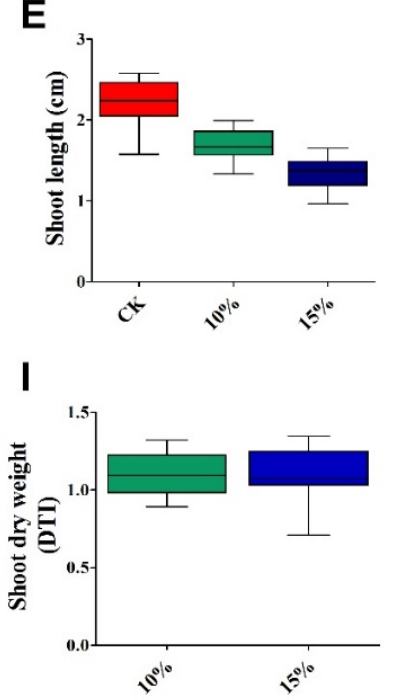

B

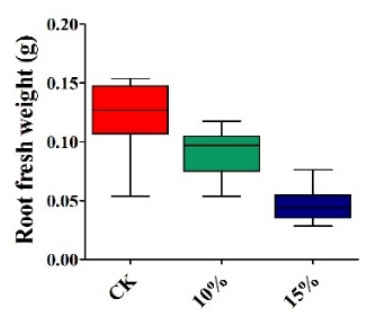

$F$
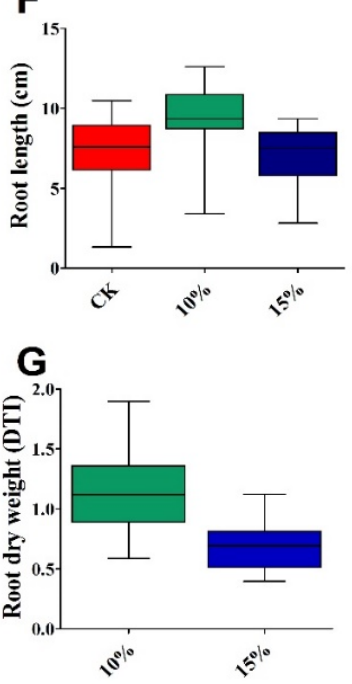

C

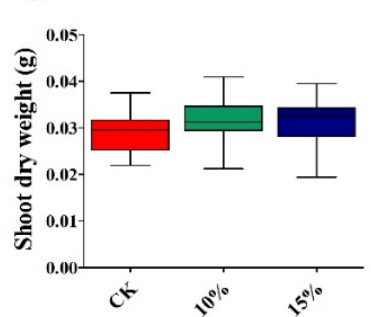

G

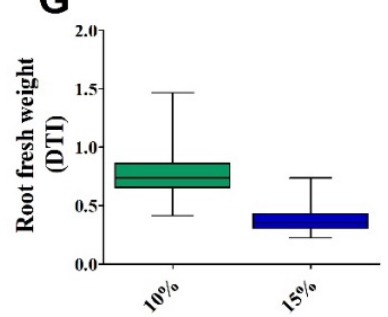

K

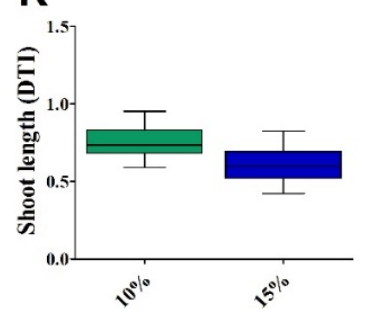

D

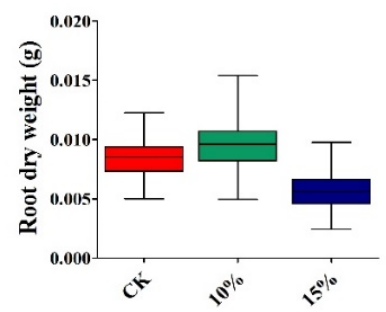

H

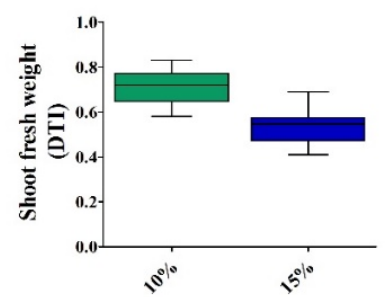

$\mathbf{L}$

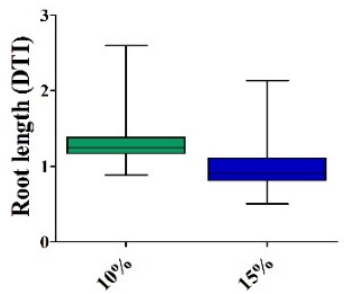

Figure 2. Box and whisker charts showing the variation of traits. (A) shoot fresh weight, (B) root fresh weight, (C) shoot dry weight, (D) root dry weight, (E) shoot length, (F) root length in rapeseed seedlings under control, 10\%, and 15\% PEG-6000 treatments. Boxplots illustrate reduction in DTI values of (G) shoot fresh weight, (H) root fresh weight, (I) shoot dry weight, (J) root dry weight, $(\mathbf{K})$ shoot length and $(\mathbf{L})$ root length under drought stress in rapeseed seedlings.

ShFW had the highest values in JYZ 158 (0.177) and FY 520 (0.181), and the lowest values in YG 2009 (0.108) and NZ 1838 (0.112) under 15\% PEG-6000. For ShDW, HYZ 62 showed a highest value of 0.039 , while the lowest value occurred in ZY 50, of 0.019, under 15\% PEG-6000 (Table 2). For RFW, the values were highest for FY 520 (0.076) and JYZ 158 (0.054), while the lowest values were obtained for YG 2009 (0.028), TYZ 283 (0.029) and NZ 1838 (0.029) under 15\% PEG-6000. The highest values of RDW were in JYZ 158 (0.0097), and the lowest values in YG 2009 (0.0024) and NZ 1838 (0.0033) under stress (15\% PEG-6000) (Table 2). Under 15\% PEG-6000, ShL was higher in FY 520 (1.466) and JYZ 158 (1.463) and lowest in QY 7 (1.193), while RL was higher in JYZ 158 (9.183) and FY 520 (8.143), and lower in YY 28 (2.817) (Table 2). Increased sensitivity of germination to PEG-6000 treatments for different cultivars was indicated by lower drought tolerance index (DTI) values. A large variation among genotypes was detected concerning their responses to drought stress. For DTI, the cultivar's mean values under 10\% PEG-6000 were 0.711, 0.752, 1.101, 1.153, 0.751 and 1.342, while under 15\% PEG-6000 the mean values were 0.532, 0.38, 1.101, 0.680, 0.600 and 0.991 for ShFW, RFW, ShDW, RDW, ShL and RL, respectively (Table 3, Figure 2). Furthermore, the drought tolerance index (DTI) values were much lower in some cultivars, including the sensitive cultivars YG 2009 and NZ 1838. 
Table 2. Seedling growth-related traits under PEG-6000 induced drought stress.

\begin{tabular}{|c|c|c|c|c|c|c|c|c|c|c|c|c|c|c|c|c|c|c|}
\hline \multirow{2}{*}{ Variety } & \multicolumn{3}{|c|}{ ShFW } & \multicolumn{3}{|c|}{ RFW } & \multicolumn{3}{|c|}{ ShDW } & \multicolumn{3}{|c|}{ RDW } & \multicolumn{3}{|c|}{$\begin{array}{l}\text { ShL } \\
\end{array}$} & \multicolumn{3}{|c|}{ RL } \\
\hline & CK & $10 \%$ & $15 \%$ & CK & $10 \%$ & $15 \%$ & CK & $10 \%$ & $15 \%$ & CK & $10 \%$ & $15 \%$ & CK & $10 \%$ & $15 \%$ & CK & $10 \%$ & $15 \%$ \\
\hline CY 81 & $0.235^{\mathrm{f}-\mathrm{i}}$ & 0.188 efg & $0.135^{g-j}$ & $0.133^{c-f}$ & $0.117 \mathrm{a}$ & $0.055 \mathrm{~cd}$ & $0.025^{\mathrm{e}-\mathrm{h}}$ & $0.030^{f-1}$ & $0.026 \mathrm{~g}$ & $0.0084^{d-g}$ & $0.0098 \mathrm{c}-\mathrm{f}$ & $0.0087 \mathrm{~b}$ & $1.965 \mathrm{hij}$ & $1.873 \mathrm{cde}$ & $1.620^{\mathrm{a}}$ & $8.691 \mathrm{bcd}$ & $9.681^{\mathrm{d}-\mathrm{g}}$ & $8.469^{a-d}$ \\
\hline YYZ3 & $0.205^{\mathrm{i}}$ & $0.129 \mathrm{~m}$ & $0.114 \mathrm{kl}$ & $0.117^{\mathrm{f}-\mathrm{i}}$ & $0.075^{\mathrm{h}-\mathrm{k}}$ & $0.050 \mathrm{def}$ & 0.024 ghi & $0.021 \mathrm{n}$ & $0.025^{\mathrm{h}}$ & $0.0082 \mathrm{fgh}$ & $0.0087 \mathrm{fg}$ & $0.00466^{\mathrm{j}}$ & $2.160^{\mathrm{f}-\mathrm{j}}$ & $1.473 \mathrm{klm}$ & $1.186^{b-f}$ & $8.450 \mathrm{bcd}$ & 10.18 def & $\begin{array}{l}7.4057 \mathrm{c}-\mathrm{f} \\
7.59\end{array}$ \\
\hline YY 28 & $0.270^{c-f}$ & 0.196 def & $0.142^{d-g}$ & $0.054 \mathrm{~m}$ & $0.064^{\mathrm{i}-1}$ & 0.040 ghi & $0.032 \mathrm{~b}$ & $0.034 \mathrm{~d}-\mathrm{j}$ & $0.033 \mathrm{~cd}$ & $0.0050^{1}$ & $0.0081 \mathrm{~g}$ & $0.0057 \mathrm{hij}$ & $2.236^{\mathrm{d}-\mathrm{h}}$ & $1.993 \mathrm{a}$ & $1.486 \mathrm{abc}$ & $1.320 \mathrm{j}$ & $3.427^{1}$ & $2.817^{1}$ \\
\hline CY 36 & $0.225 \mathrm{hi}$ & $0.182^{\mathrm{f}-\mathrm{i}}$ & $0.155 \mathrm{~d}-\mathrm{f}$ & $0.106^{h-k}$ & 0.094 efg & $0.059 \mathrm{bc}$ & $0.027 \mathrm{cde}$ & 0.032 ge-k & $0.033 \mathrm{~cd}$ & $0.0091 \mathrm{def}$ & 0.0096 efg & 0.0075 bc & $2.007 \mathrm{~g}-\mathrm{j}$ & 1.729 def & $\begin{array}{l}1.4000 \mathrm{ab} \\
1.482 \mathrm{ab}\end{array}$ & $5.987 \mathrm{fgh}$ & $9.250 \mathrm{fgh}$ & $7.689 \mathrm{~b}-\mathrm{e}$ \\
\hline ZY 50 & 0.228 ghi & $0.176 \mathrm{~g}-\mathrm{j}$ & $0.118^{\mathrm{h}-1}$ & 0.106 ijk & 0.098 cde & $0.0355^{\mathrm{ij}}$ & $0.032 \mathrm{~b}$ & $0.031 \mathrm{f}-1$ & $0.033 \mathrm{~cd}$ & $0.0068^{\text {hij }}$ & $0.0128 \mathrm{~b}$ & $0.0046 \mathrm{kl}$ & $1.883 \mathrm{ij}$ & $1.613^{\mathrm{f}-\mathrm{j}}$ & $\begin{array}{l}1.450 \mathrm{a}-\mathrm{d} \\
1.450\end{array}$ & $7.563^{\mathrm{c}-\mathrm{f}}$ & $10.91 \mathrm{bcd}$ & $\begin{array}{l}7.1053 \mathrm{~d}-\mathrm{h} \\
60.83 \mathrm{~d}\end{array}$ \\
\hline QY 33 & $\begin{array}{l}0.278 \mathrm{cde} \\
0.274\end{array}$ & $0.159 \mathrm{jl}$ & $0.115 \mathrm{jkl}$ & $0.124^{f-1}$ & $0.081 \mathrm{fgh}$ & $0.061 \mathrm{abc}$ & 0.024 ghi & $0.024 \mathrm{mn}$ & $0.019^{\mathrm{i}}$ & $0.0081 \mathrm{e}-\mathrm{h}$ & $0.0107 \mathrm{~cd}$ & $\begin{array}{l}0.006063^{2} \mathrm{gh} \\
0.0\end{array}$ & $2.363 \mathrm{a}-\mathrm{d}$ & $1.397 \mathrm{mn}$ & $\begin{array}{l}1.4226^{\mathrm{a}-\mathrm{f}} \\
\text { 1 }\end{array}$ & $9.020 \mathrm{abc}$ & $12.62 \mathrm{a}$ & $8.063^{6} \mathrm{~b}-\mathrm{e}$ \\
\hline ZY 51 & $\begin{array}{l}0.274 \text { cde } \\
0.07\end{array}$ & $0.183 \mathrm{fgh}$ & 0.158 b-e & $\begin{array}{l}0.1244 \mathrm{ab} \\
0.147 \mathrm{ab}\end{array}$ & $0.098 \mathrm{de}$ & 0.049 def & $\begin{array}{l}0.024 \mathrm{obc} \\
0.030 \mathrm{bc}\end{array}$ & $0.036^{b-e}$ & $0.038 \mathrm{ab}$ & $0.0107 \mathrm{bc}$ & $0.0095^{\mathrm{c}-\mathrm{g}}$ & $\begin{array}{l}0.00055^{\circ} \mathrm{ij} \\
0.050\end{array}$ & 2.353 a-e & $1.733 \mathrm{~d}-\mathrm{h}$ & $1.536 \mathrm{ab}$ & $6.587 \mathrm{e}-\mathrm{h}$ & $8.481 \mathrm{hi}$ & $5.9844^{\mathrm{f}-\mathrm{i}}$ \\
\hline XZY 518 & $0.243 \mathrm{e}-\mathrm{h}$ & $0.1655^{-1}-1$ & $\begin{array}{l}0.1147^{c} \mathrm{c} \\
0\end{array}$ & $0.138^{b}-\mathrm{b}$ & $0.105^{a-e}$ & $0.054 \mathrm{~cd}$ & $0.032 \mathrm{~b}$ & $0.030 \mathrm{~h}-1$ & $\begin{array}{l}0.058 \\
0.034 a b\end{array}$ & 0.009 def & 0.0109 cde & $\begin{array}{l}0.000544^{7} \\
0.0074\end{array}$ & $1.877 \mathrm{jk}$ & $1.346^{\mathrm{n}}$ & $\begin{array}{l}1.536 \mathrm{~b}-\mathrm{f} \\
1.193 \mathrm{~b}\end{array}$ & 7.621 cde & $\begin{array}{l}8.4851 \mathrm{fh} \\
9.353 \text { ghh }\end{array}$ & $6.637 \mathrm{e}^{-\mathrm{h}}$ \\
\hline GHY 8 & $0.288 \mathrm{bc}$ & $0.162^{-1-i}$ & $0.118^{\mathrm{i}-1}$ & $0.098 \mathrm{kl}$ & 0.077 ghi & $\begin{array}{l}0.054 \mathrm{huh} \\
0.039 \mathrm{ghi}\end{array}$ & $\begin{array}{l}0.032 \text { cde } \\
0.026 \text { e }\end{array}$ & $0.029 \mathrm{jkl}$ & $\begin{array}{l}0.0344^{0} \\
0.027\end{array}$ & $0.0099 \mathrm{~cd}$ & $0.0058 \mathrm{~h}$ & $0.0046 \mathrm{~lm}$ & $2.576^{\mathrm{a}}$ & $\begin{array}{l}1.1840 \mathrm{ccd} \\
1886 \mathrm{bcd}\end{array}$ & $1087 \mathrm{f}$ & $5.563 \mathrm{~h}$ & . $68553 \mathrm{j}$ & $\begin{array}{l}6.6377 \mathrm{e}-\mathrm{h} \\
6.65 \mathrm{e}\end{array}$ \\
\hline ZYZ 108 & $\begin{array}{l}0.288 \mathrm{a} \\
0.342 \mathrm{a}\end{array}$ & $0.238 \mathrm{a}$ & $0.158 \mathrm{a}-\mathrm{d}$ & . & $0.098 \mathrm{de}$ & 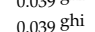 & $\begin{array}{c}0.026 \text { a } \\
0.037\end{array}$ & $\begin{array}{l}0.02971 \mathrm{a} \\
0.041\end{array}$ & $0.026 \mathrm{fg}$ & $0.0050 \mathrm{kl}$ & $\begin{array}{r}0.00088^{\mathrm{c}-\mathrm{g}} \\
0.009\end{array}$ & $0.0046 \mathrm{~lm}$ & $2.566 \mathrm{a}$ & $1.8686 \mathrm{e}-\mathrm{i}$ & $\begin{array}{l}1.2087 \\
1203-\mathrm{f}\end{array}$ & $\begin{array}{l}5.5653 \\
3.911\end{array}$ & $\begin{array}{l}6.8577 \mathrm{fgh} \\
9371 \mathrm{ghh}\end{array}$ & $\begin{array}{l}6.6517 \\
6551 \mathrm{e}-\mathrm{h}\end{array}$ \\
\hline $\begin{array}{l}\text { ZYZ } 108 \\
\text { NZ }\end{array}$ & $\begin{array}{l}0.342^{\mathrm{a}} \\
0.26 \mathrm{c}^{-\mathrm{g}}\end{array}$ & $\begin{array}{l}0.238^{\mathrm{a}} \\
0.16 \mathrm{i}^{-1}\end{array}$ & $\begin{array}{l}0.155^{\mathrm{d}-a} \\
0.112 \mathrm{kl}\end{array}$ & $\begin{array}{l}0.0677^{j} \\
0.11 \mathrm{k}\end{array}$ & $\begin{array}{l}0.098 \text { ene } \\
0.079 \text { gh }\end{array}$ & $\begin{array}{c}0.039 \mathrm{~g}^{0.1 \mathrm{ul}} \\
0.029 \mathrm{j}\end{array}$ & $\begin{array}{l}0.037 \mathrm{a} \\
0.031 \mathrm{~b}\end{array}$ & $\begin{array}{l}0.041^{\mathrm{a}} \\
0.03 \mathrm{i}^{-1}\end{array}$ & $\begin{array}{l}0.0266^{0.8} \\
0.031 \mathrm{gh}\end{array}$ & $\begin{array}{l}0.00000^{\mathrm{K}} \\
0.0062 \mathrm{jk}\end{array}$ & $\begin{array}{l}0.00944^{\circ} \\
0.0055\end{array}$ & $\begin{array}{l}0.000466^{m i n} \\
0.0033^{n}\end{array}$ & $\begin{array}{l}2.5666^{\mathrm{a}} \\
2.003 \mathrm{~g}\end{array}$ & $\begin{array}{l}1.6866^{6-17} \\
1.56 \mathrm{k} l\end{array}$ & $\begin{array}{l}1.203066^{0-1} \\
0.96 f\end{array}$ & $\begin{array}{r}3.9111^{1} \\
7.357 \mathrm{~d}-\mathrm{g}\end{array}$ & $\begin{array}{l}9.3711171 \\
9.317 \mathrm{fgh}\end{array}$ & $\begin{array}{l}6.517^{-10} \\
{ }_{3} .717 \\
\mathrm{kll}\end{array}$ \\
\hline $\begin{array}{c}x+553 \\
\text { YY } 9\end{array}$ & $\begin{array}{l}0.288^{\mathrm{c}-\mathrm{g}} \\
0.2 \mathrm{c}^{2}\end{array}$ & $0.2228 \mathrm{kl}$ & $0.129 \mathrm{~g}-\mathrm{k}$ & 0.129 def & 0.0541 & $\begin{array}{l}0.0444 \\
0.035 \text { hij }\end{array}$ & $0.031^{\mathrm{b}}$ & $0.033 \mathrm{~d}-\mathrm{i}$ & 0.032 cde & 0.0073 hij & $0.0054^{\circ} \mathrm{h}$ & $0.0037 \mathrm{mn}$ & $2.166^{d-h}$ & $1.473 \mathrm{~lm}$ & $1.049 \mathrm{c}-\mathrm{f}$ & $5.993 \mathrm{gh}$ & $5.877 \mathrm{k}$ & $3.463 \mathrm{kl}$ \\
\hline HYZ 62 & $0.354^{\mathrm{a}}$ & $\begin{array}{l}0.158 \mathrm{ab} \\
0.227 \mathrm{ab}\end{array}$ & $0.164 \mathrm{abc}$ & $0.147 \mathrm{ab}$ & $\begin{array}{l}0.0 .04^{\mathrm{a}} \\
0.15^{\mathrm{a}}\end{array}$ & $\begin{array}{l}0.0335 \\
0.035 \\
\text { ij }\end{array}$ & $0.029 \mathrm{bcd}$ & $0.036 \mathrm{bcd}$ & $\begin{array}{l}0.03259 \mathrm{a} \\
0.039\end{array}$ & 0.0123 a & $0.0098^{c-f}$ & $0.0057 \mathrm{ghi}$ & $2.316^{\mathrm{b}-\mathrm{g}}$ & $1.573^{\mathrm{h}-1}$ & $1.286^{\mathrm{a}-\mathrm{e}}$ & $7.583^{b-e}$ & $8.597 \mathrm{ghi}$ & 5.573 hij \\
\hline $\begin{array}{l}\text { T11L 62 } \\
\text { QY } 3\end{array}$ & $0.261^{\mathrm{c}-\mathrm{g}}$ & $\begin{array}{l}0.227 \\
0.175 \\
\mathrm{~g}-\mathrm{k}\end{array}$ & $\begin{array}{l}0.164 \mathrm{w}-\mathrm{g} \\
0.142 \mathrm{~d}\end{array}$ & $\begin{array}{l}0.147 \mathrm{a} \\
0.149 \mathrm{ab}\end{array}$ & 0.113 abc & $\begin{array}{l}0.0355^{3} \\
0.064 \mathrm{ab}\end{array}$ & $0.028 \mathrm{def}$ & $\begin{array}{l}0.00268 \mathrm{~lm} \\
0.02\end{array}$ & 0.030 ef & 0.0013 def & $0.0097 \mathrm{c}^{\mathrm{c}-\mathrm{f}}$ & 0.0064 ef & $2.226^{\mathrm{d}-\mathrm{b}}$ & $1.566 \mathrm{jkl}$ & $1.407 \mathrm{a}-\mathrm{e}$ & $9.251 \mathrm{ab}$ & $\begin{array}{l}8.579 .19 \mathrm{a} \\
12 .\end{array}$ & $\begin{array}{r}5.4873 \mathrm{a}-\mathrm{d} \\
8 \mathrm{~d}\end{array}$ \\
\hline QY 7 & $0.206^{\mathrm{i}}$ & $0.156^{1}$ & $0.142^{\mathrm{e}-\mathrm{h}}$ & $0.113 \mathrm{~g}-\mathrm{j}$ & $0.074^{\mathrm{h}-\mathrm{k}}$ & $0.044 \mathrm{fg}$ & $0.022 \mathrm{i}$ & $0.029 \mathrm{klm}$ & $0.027^{\mathrm{fg}}$ & $0.0080^{--h}$ & $0.0091^{d-g}$ & 0.0051 ijk & $1.576 \mathrm{k}$ & $1.333^{n}$ & $1.193^{\mathrm{a}-\mathrm{f}}$ & $7.567 \mathrm{def}$ & $9.260 \mathrm{fgh}$ & $8.491^{\mathrm{a}-\mathrm{d}}$ \\
\hline $\mathrm{ZS} 11$ & $0.244^{\mathrm{d}-\mathrm{h}}$ & 0.188 efg & $0.135 \mathrm{f}-\mathrm{i}$ & $0.125^{\mathrm{d}-\mathrm{g}}$ & $0.115 \mathrm{ab}$ & $0.041 \mathrm{gh}$ & $0.025 \mathrm{fgh}$ & $0.030 \mathrm{~g}-1$ & $0.031 \mathrm{de}$ & $0.0087 \mathrm{~d}-\mathrm{g}$ & $0.0097 \mathrm{c}-\mathrm{f}$ & $0.0068 \mathrm{de}$ & $2.353^{\mathrm{a}-\mathrm{f}}$ & $1.580 \mathrm{~g}-\mathrm{k}$ & $1.653 \mathrm{ab}$ & $7.397 \mathrm{~d}-\mathrm{g}$ & $9.38 \mathrm{e}^{\mathrm{e}-\mathrm{h}}$ & $9.070 \mathrm{abc}$ \\
\hline YG 2009 & $0.238^{\mathrm{f}-\mathrm{i}}$ & $0.158 \mathrm{j} \mathrm{kl}$ & 0.1081 & $0.086^{1}$ & $0.062^{j} \mathrm{kl}$ & $0.028^{\mathrm{j}}$ & $0.027 \mathrm{def}$ & $0.031^{\mathrm{f}-1}$ & 0.032 cde & $0.00622^{\mathrm{j} k}$ & $0.0054 \mathrm{~h}$ & $0.0024^{\circ}$ & $2.241^{\mathrm{d}-\mathrm{g}}$ & $1.581^{\mathrm{i}-1}$ & $1.007 \mathrm{def}$ & $5.876 \mathrm{gh}$ & $7.437 \mathrm{ij}$ & 4.613 jik \\
\hline HYZ 72 & $0.285^{\mathrm{C}}$ & $0.226 \mathrm{abc}$ & $0.142^{f-i}$ & $0.124 \mathrm{e}-\mathrm{h}$ & $0.059 \mathrm{kl}$ & 0.035 hi & $0.031 \mathrm{~b}$ & $0.038 \mathrm{ab}$ & $0.036^{\mathrm{b}}$ & 0.0075 ghi & $0.0107^{\mathrm{c}}$ & $0.0052 \mathrm{sjk}$ & $2.493 \mathrm{abc}$ & $1.961 \mathrm{ab}$ & 1.399 a-e & 8.460 bcd & $12.097 \mathrm{ab}$ & $7.393 \mathrm{~d}-\mathrm{g}$ \\
\hline QY1 & $0.221 \mathrm{hi}$ & $0.168^{\mathrm{h}-1}$ & $0.143^{d-g}$ & $0.152 \mathrm{ab}$ & $0.102^{b-e}$ & $0.054 \mathrm{de}$ & 0.025 efg & $0.029^{\mathrm{i}-1}$ & $0.033 \mathrm{~cd}$ & 0.009 def & $0.0127 \mathrm{~b}$ & $0.0063 \mathrm{fg}$ & $2.156^{\mathrm{f}-\mathrm{i}}$ & $\begin{array}{l}1.901 \\
1.678 \mathrm{e}-\mathrm{j}\end{array}$ & $1.573 \mathrm{ab}$ & 9.103 ab & 10.65 cde & $9.357 \mathrm{a}^{\circ}$ \\
\hline TYZ 283 & $0.248 \mathrm{~d}-\mathrm{h}$ & 0.206 sde & $0.147^{c-g}$ & $0.128^{d-g}$ & 0.075 hij & $0.029 \mathrm{j}$ & $0.032 \mathrm{~b}$ & $0.037 \mathrm{abc}$ & $0.037 \mathrm{ab}$ & $0.0081 \mathrm{e}-\mathrm{h}$ & $0.0098^{c-f}$ & $0.0038 \mathrm{mn}$ & $2.166^{\mathrm{e}-\mathrm{i}}$ & $1.837 \mathrm{cde}$ & $1.317^{\text {a-e }}$ & $8.481 \mathrm{bcd}$ & 9.9.993 def & 5.707 ghi \\
\hline GZ1 & $0.277 \mathrm{~cd}$ & $0.206 \mathrm{cde}$ & $0.156^{\mathrm{b}-\mathrm{f}}$ & $0.141^{\mathrm{a}-\mathrm{d}}$ & $0.113^{\mathrm{a}-\mathrm{s}}$ & $\begin{array}{l}0.049 \text { efg } \\
0.044 \text { e }\end{array}$ & $0.023 \mathrm{hi}$ & $0.030 \mathrm{~g}-1$ & $0.031 \mathrm{de}$ & $0.0095 \mathrm{cde}$ & $0.0124^{\circ} \mathrm{b}$ & $0.0067 \mathrm{de}$ & $2.576^{\mathrm{a}}$ & $1.967 \mathrm{abc}$ & $1.350^{\mathrm{a}-\mathrm{d}}$ & $9.169 \mathrm{ab}$ & $12.0 \mathrm{ab}$ & $9.323 \mathrm{ab}$ \\
\hline FY 520 & $0.332 \mathrm{a}$ & $0.237 \mathrm{a}$ & $0.181 \mathrm{ab}$ & $0.148 \mathrm{abc}$ & 0.095 ef & $0.076 \mathrm{a}$ & $0.032 \mathrm{~b}$ & $0.034^{\mathrm{c}-\mathrm{h}}$ & $0.037 \mathrm{ab}$ & $0.0086 \mathrm{~d}-\mathrm{h}$ & $\begin{array}{l}0.0082 \mathrm{~g} \\
0.014\end{array}$ & 0.0065 ef & $2.580 \mathrm{a}$ & $1.746^{\mathrm{d}-\mathrm{g}}$ & $1.466 \mathrm{abc}$ & $10.32 \mathrm{a}$ & $\begin{array}{l}9.147 \\
\mathrm{fghh}\end{array}$ & $8.143 \mathrm{c}-\mathrm{f}$ \\
\hline Mean & 0.266 & 0.188 & 0.140 & 0 & 0.090 & 0.045 & 0.089 & 0.032 & 0.031 & 0.0084 & 0.0094 & 0.0056 & 2.235 & 1.672 & $\begin{array}{l}1.400 \\
1.337\end{array}$ & 7.470 & 9.463 & 6.968 \\
\hline
\end{tabular}


Table 3. Drought tolerance index of germination and seedling growth traits of rapeseed cultivars under drought stress.

\begin{tabular}{|c|c|c|c|c|c|c|c|c|c|c|c|c|c|c|c|c|c|c|c|c|}
\hline \multirow{2}{*}{ Variety } & \multicolumn{2}{|c|}{ FG\% } & \multicolumn{2}{|c|}{ GR } & \multicolumn{2}{|c|}{ VI (II) } & \multicolumn{2}{|c|}{ VI (I) } & \multicolumn{2}{|c|}{ ShFW } & \multicolumn{2}{|c|}{ RFW } & \multicolumn{2}{|c|}{ ShDW } & \multicolumn{2}{|c|}{ RDW } & \multicolumn{2}{|c|}{ ShL } & \multicolumn{2}{|c|}{ RL } \\
\hline & $10 \%$ & $15 \%$ & $10 \%$ & $15 \%$ & $10 \%$ & $15 \%$ & $10 \%$ & $15 \%$ & $10 \%$ & $15 \%$ & $10 \%$ & $15 \%$ & $10 \%$ & $15 \%$ & $10 \%$ & $15 \%$ & $10 \%$ & $15 \%$ & $10 \%$ & $15 \%$ \\
\hline CY 81 & $0.98 \mathrm{~cd}$ & $0.63 \mathrm{k}$ & $0.99 \mathrm{~b}$ & $0.47 \mathrm{kl}$ & $0.82^{\mathrm{a}}$ & $0.32 \mathrm{~m}$ & $1.07 \mathrm{gh}$ & $0.59^{\mathrm{i}}$ & $0.80 \mathrm{ab}$ & $0.57 \mathrm{cde}$ & $0.88^{\mathrm{C}}$ & $0.41 \mathrm{de}$ & $1.18 \mathrm{cde}$ & $1.06 \mathrm{fg}$ & $1.16^{\mathrm{d}}$ & $0.95^{\mathrm{b}}$ & $0.95^{\mathrm{a}}$ & $0.82 \mathrm{a}$ & $1.11^{\mathrm{i}}$ & $0.97 \mathrm{ef}$ \\
\hline YYZ3 & $0.99 \mathrm{bcd}$ & $1.00^{a}$ & $0.74 \mathrm{ghi}$ & $0.65 \mathrm{~cd}$ & $0.62^{\mathrm{i}}$ & $0.50^{\mathrm{b}}$ & $1.09 \mathrm{e}-\mathrm{h}$ & $0.82^{e f}$ & $0.63 \mathrm{jkl}$ & $0.56 \mathrm{def}$ & $0.64^{\mathrm{ij}}$ & $0.42 \mathrm{de}$ & $0.89 \mathrm{k}$ & $1.05 \mathrm{fg}$ & $1.066^{\mathrm{d}}$ & $0.56^{\mathrm{k}}$ & $0.68^{\mathrm{f}-\mathrm{i}}$ & $0.55^{\mathrm{ij}}$ & $1.20 \mathrm{ghi}$ & $0.89 \mathrm{fg}$ \\
\hline YY 28 & $0.98 \mathrm{~cd}$ & $0.80^{\mathrm{i}}$ & $0.78^{\mathrm{efg}}$ & $0.40 \mathrm{mn}$ & $0.78 \mathrm{ab}$ & 0.45 efg & $1.49^{\mathrm{b}}$ & $0.96^{\mathrm{bc}}$ & $0.72^{\mathrm{d}-\mathrm{h}}$ & $0.52^{f-j}$ & $1.19^{\mathrm{b}}$ & $0.74^{\mathrm{a}}$ & $1.07^{\mathrm{fgh}}$ & $1.06 \mathrm{gh}^{\mathrm{gh}}$ & $1.61^{\mathrm{b}}$ & $1.12^{\mathrm{a}}$ & $0.89^{\mathrm{b}}$ & $0.66 \mathrm{de}$ & $2.59^{\mathrm{a}}$ & $2.13 \mathrm{a}$ \\
\hline CY 36 & $0.99 \mathrm{bcd}$ & 0.89 efg & $0.90 \mathrm{~d}$ & $0.75^{\mathrm{b}}$ & $0.82^{\mathrm{a}}$ & $0.58 \mathrm{a}$ & $1.36^{\mathrm{c}}$ & $1.03 \mathrm{ab}$ & $0.81 \mathrm{ab}$ & $0.68^{\mathrm{a}}$ & $0.88^{\mathrm{c}}$ & $0.55^{\mathrm{b}}$ & $1.20 \mathrm{bcd}$ & $1.23 \mathrm{c}^{\mathrm{de}}$ & $0.98 \mathrm{ef}$ & $0.82 \mathrm{de}$ & $0.86^{\mathrm{b}}$ & $0.74 \mathrm{bc}$ & $1.54^{\mathrm{c}}$ & $1.28^{\mathrm{c}}$ \\
\hline JYZ 158 & $1.02 \mathrm{abc}$ & $1.01^{\mathrm{a}}$ & $0.76^{\text {tgh }}$ & $0.60 \mathrm{efg}$ & $0.75 \mathrm{bcd}$ & $0.49 \mathrm{bc}$ & $1.08 \mathrm{fgh}$ & 0.83 ef & $0.73^{\mathrm{d}-\mathrm{g}}$ & $0.55^{\mathrm{e}-\mathrm{h}}$ & 0.76 def & $0.35^{\mathrm{fg}}$ & $0.93^{\mathrm{jk}}$ & $0.90^{\mathrm{i}}$ & $1.37^{\mathrm{c}}$ & $0.86^{\mathrm{cd}}$ & 0.74 cde & $0.58 \mathrm{ghi}$ & $1.13^{\mathrm{i}}$ & $0.87 \mathrm{gh}$ \\
\hline ZY 50 & $1.00^{a-d}$ & $0.98 \mathrm{abc}$ & $0.97 \mathrm{bc}$ & $0.79 \mathrm{ab}$ & $0.82^{\mathrm{a}}$ & 0.44 efg & $1.33^{c}$ & $0.85^{\mathrm{e}}$ & $0.77 \mathrm{bcd}$ & $0.522^{g-j}$ & $0.92^{\mathrm{c}}$ & 0.33 ghi & $0.97^{\mathrm{h}-\mathrm{k}}$ & $1.04 \mathrm{gh}$ & $1.89^{\mathrm{a}}$ & $0.68^{\mathrm{j}}$ & $0.85^{\mathrm{b}}$ & $0.76^{\mathrm{b}}$ & $1.44 \mathrm{~cd}$ & $0.90 \mathrm{fg}$ \\
\hline QY 33 & $1.03 \mathrm{ab}$ & $0.88^{\mathrm{e}-\mathrm{h}}$ & 0.78 efg & $0.48 \mathrm{ghi}$ & $0.62^{\mathrm{i}}$ & $0.39^{\mathrm{jk}}$ & $1.27 \mathrm{~cd}$ & $0.72 \mathrm{~g}$ & $0.58^{1}$ & $0.42 \mathrm{mn}$ & $0.65^{\mathrm{hij}}$ & $0.49^{\mathrm{C}}$ & $0.99 g^{g-j}$ & $0.81^{\mathrm{i}}$ & $1.32^{\mathrm{C}}$ & $0.77^{\mathrm{hi}}$ & $0.59 \mathrm{j}$ & $0.52^{\mathrm{jk}}$ & $1.39 \mathrm{~d}^{\mathrm{ef}}$ & $0.89 \mathrm{fg}$ \\
\hline ZY 51 & $0.99 \mathrm{bcd}$ & $0.70^{\mathrm{j}}$ & $0.98 \mathrm{~b}$ & 0.55 hij & $0.66^{\mathrm{hi}}$ & $0.34 \mathrm{~lm}$ & $1.13 \mathrm{e}-\mathrm{h}$ & $0.58^{\mathrm{i}}$ & $0.66^{\mathrm{ij}}$ & $0.57 \mathrm{cde}$ & $0.66 \mathrm{hi}$ & $0.33 \mathrm{gh}$ & $1.23 \mathrm{bcd}$ & $1.27 \mathrm{bcd}$ & 0.89 fgh & $0.50 \mathrm{~lm}$ & $0.73^{\mathrm{c}-\mathrm{f}}$ & 0.65 def & $1.28 \mathrm{fgh}$ & $0.90 \mathrm{fg}$ \\
\hline XZY 518 & $1.00 \mathrm{a}-\mathrm{d}$ & $0.92 \mathrm{de}$ & $0.76 \mathrm{fgh}$ & $0.54 \mathrm{hij}$ & $0.71^{\mathrm{c}-\mathrm{h}}$ & $0.48 \mathrm{bcd}$ & $1.12 \mathrm{e}-\mathrm{h}$ & $0.75 \mathrm{~g}$ & $0.68 \mathrm{~g}-\mathrm{j}$ & $0.60^{c}$ & $0.76^{\mathrm{d}-\mathrm{g}}$ & $0.39 \mathrm{ef}$ & $0.95 \mathrm{jk}$ & $1.08 \mathrm{fg}$ & $1.21 \mathrm{~d}$ & $0.82 \mathrm{ef}$ & $0.72 \mathrm{~d}-\mathrm{h}$ & 0.63 efg & $1.22 \mathrm{ghi}$ & $0.87 \mathrm{gh}$ \\
\hline GHY 8 & $1.00^{a-d}$ & $0.99 \mathrm{ab}$ & $1.00^{\mathrm{b}}$ & $0.83 \mathrm{a}$ & $0.67 \mathrm{ghi}$ & $0.40^{\mathrm{hij}}$ & $1.07 \mathrm{fgh}$ & $0.94^{\mathrm{C}}$ & $0.63^{\mathrm{kl}}$ & $0.41^{\mathrm{n}}$ & $0.78 \mathrm{def}$ & 0.39 ef & $1.09 \mathrm{fg}$ & $1.03 \mathrm{gh}$ & $0.58^{\mathrm{j}}$ & $0.46^{n}$ & $0.73^{c-g}$ & $0.42^{\mathrm{n}}$ & $1.23 \mathrm{gh}$ & $1.19^{\mathrm{c}}$ \\
\hline ZYZ 108 & $0.96 \mathrm{de}$ & $0.87^{\mathrm{fgh}}$ & 0.74 ghi & $0.52^{\mathrm{ij}}$ & $0.79 \mathrm{ab}$ & $0.42 \mathrm{~g}-\mathrm{j}$ & $1.65 \mathrm{a}$ & $1.04 \mathrm{a}$ & $0.69 \mathrm{f}-\mathrm{i}$ & $0.46 \mathrm{kl}$ & $1.46^{\mathrm{a}}$ & $0.59 \mathrm{~b}$ & $1.09 \mathrm{fg}$ & $0.70^{\mathrm{j}}$ & $1.87^{\mathrm{a}}$ & $0.91 \mathrm{bc}$ & $0.66^{\mathrm{i}}$ & $0.47 \mathrm{Imn}$ & $2.39 \mathrm{~b}$ & $1.66^{\mathrm{b}}$ \\
\hline $\begin{array}{l}\text { XZY } 553 \\
\end{array}$ & $1.00^{\mathrm{a}-\mathrm{d}}$ & $\begin{array}{l}0.61 \mathrm{abc} \\
0.98\end{array}$ & $0.74 \mathrm{ghi}^{\mathrm{hi}}$ & $\begin{array}{l}0.42 \\
0.58 \mathrm{fgh}\end{array}$ & $0.74 \mathrm{~b}-\mathrm{f}$ & $0.42 \mathrm{~g}-\mathrm{j}$ & $1.09 \mathrm{fgh}$ & $0.94 \mathrm{~cd}$ & $0.77 \mathrm{bcd}$ & $0.51 \mathrm{hij}$ & $0.69 \mathrm{ghi}$ & $0.29^{\mathrm{hij}}$ & $\begin{array}{l}0.94 \mathrm{fgh} \\
1.03 \mathrm{fgh}\end{array}$ & $\begin{array}{l}0.93 \mathrm{fg} \\
1.03\end{array}$ & $\begin{array}{c}0.806^{\mathrm{i}} \\
0\end{array}$ & $\begin{array}{l}0.54 \\
0.51 \mathrm{~mm}\end{array}$ & $0.71 \mathrm{~d}-\mathrm{h}$ & 0.65 def & $1.20 \mathrm{hi}$ & 1.05 de \\
\hline YY 9 & $0.97 \mathrm{de}$ & $0.78^{\mathrm{i}}$ & $\begin{array}{c}.0 .4 \mathrm{~g} \\
0.70^{\mathrm{i}}\end{array}$ & $0.47 \mathrm{kl}$ & $\begin{array}{r}.0 .52 \mathrm{j} \\
\end{array}$ & $\begin{array}{l}.428 \\
0.33 \mathrm{~m}\end{array}$ & $\begin{array}{r}0.87^{\mathrm{i}} \\
\end{array}$ & $0.43{ }^{3}$ & $0.60 \mathrm{kl}$ & $0.49 \mathrm{jk}$ & $0.41 \mathrm{k}$ & $\begin{array}{l}0.27 \mathrm{jk} \\
0.27\end{array}$ & $1.07 \mathrm{fgh}$ & $\begin{array}{l}1.03 \\
1.01 \mathrm{gh}\end{array}$ & $0.74^{\mathrm{i}}$ & $0.50^{1}$ & $0.6 \mathrm{f}^{\mathrm{fi}}$ & $0.48 \mathrm{klm}$ & $0.98^{j}$ & $0.57 \mathrm{k}$ \\
\hline HYZ 62 & $1.01^{\mathrm{a}-\mathrm{d}}$ & $0.91 \mathrm{def}$ & $0.72 \mathrm{hi}$ & $0.47 \mathrm{kl}$ & $0.67 \mathrm{ghi}$ & $0.36 \mathrm{kl}$ & $1.04^{\mathrm{h}}$ & $0.63 \mathrm{hi}$ & $0.64^{\mathrm{jk}}$ & $0.46 \mathrm{kl}$ & $0.71^{-\mathrm{fi}}$ & $0.24 \mathrm{kl}$ & $1.25 \mathrm{bc}$ & $1.34^{\mathrm{a}}$ & $0.79 \mathrm{hi}$ & $0.46^{n}$ & $0.68 \mathrm{ghi}$ & 0.55 hij & $1.13^{\mathrm{i}}$ & $0.73^{\mathrm{ij}}$ \\
\hline QY3 & $0.98 \mathrm{~cd}$ & $0.86 \mathrm{gh}$ & 0.74 ghi & $0.36^{n}$ & 0.69 fgh & $0.4 \mathrm{e}^{\mathrm{e}-\mathrm{h}}$ & $1.18 \mathrm{def}$ & $0.74 \mathrm{~g}$ & $0.67^{\text {hij }}$ & $0.54^{-\mathrm{e}}$ & $0.75 \mathrm{~d}-\mathrm{g}$ & $0.43 \mathrm{de}$ & $0.97 \mathrm{ghi}$ & $1.05 \mathrm{ef}$ & $1.08 \mathrm{de}$ & $0.71^{\mathrm{i}}$ & $0.70^{--i}$ & 0.63 efg & $1.32 \mathrm{efg}$ & $0.92 \mathrm{fg}$ \\
\hline QY 7 & $1.00^{\mathrm{a}-\mathrm{d}}$ & $1.00 \mathrm{a}$ & $0.76 \mathrm{fgh}$ & $0.68^{c}$ & $0.72^{c-g}$ & $0.58 \mathrm{a}$ & $1.16 \mathrm{efg}$ & $1.05 \mathrm{a}$ & $0.75^{b-e}$ & $0.68^{\mathrm{a}}$ & $0.65 \mathrm{hig}$ & $0.39 \mathrm{ef}$ & $1.32^{\mathrm{a}}$ & $1.25 \mathrm{ab}$ & $1.12 \mathrm{~d}$ & $0.63 \mathrm{k}$ & $0.84 \mathrm{~b}$ & $0.75 \mathrm{~b}$ & $\begin{array}{l}1.52 \\
1.22 \mathrm{ghi}\end{array}$ & $1.12 \mathrm{~d}$ \\
\hline ZS 11 & $1.02 \mathrm{abc}$ & $0.97 \mathrm{abc}$ & 0.81 ef & $0.81^{\mathrm{a}}$ & $0.83^{\mathrm{a}}$ & 0.46 cde & $1.14 \mathrm{efg}$ & $1.07^{\mathrm{a}}$ & $0.77 \mathrm{bcd}$ & $0.55 \mathrm{efg}$ & $0.91 \mathrm{c}$ & $0.32 \mathrm{ghi}$ & $1.23 \mathrm{ab}$ & $1.26 \mathrm{ab}$ & $1.12 \mathrm{~d}$ & $0.78 \mathrm{fg}$ & $0.07 \mathrm{hi}$ & $0.70 \mathrm{~cd}$ & $1.26 \mathrm{gh}$ & $1.22^{\mathrm{c}}$ \\
\hline YG 2009 & $1.00^{a-d}$ & $0.43^{1}$ & $1.12^{\mathrm{a}}$ & $0.49 \mathrm{jk}$ & $0.67 \mathrm{ghi}$ & $0.18^{\circ}$ & $1.111^{\mathrm{e}-\mathrm{h}}$ & $0.30^{\mathrm{k}}$ & $0.66^{\mathrm{ij}}$ & $0.45 \mathrm{~lm}$ & $0.71 \mathrm{e}-\mathrm{h}$ & $0.32 \mathrm{ghi}$ & $1.17 \mathrm{cde}$ & $1.20^{\mathrm{c}-\mathrm{e}}$ & $0.80 \mathrm{hi}$ & $0.39^{\circ}$ & $0.70 \mathrm{~d}-\mathrm{i}$ & $0.44 \mathrm{mn}$ & $1.26 \mathrm{gh}$ & $0.78 \mathrm{hi}$ \\
\hline HYZ 72 & $1.04 \mathrm{a}$ & $0.94 \mathrm{~cd}$ & $0.91 \mathrm{~d}$ & 0.64 cde & $0.72^{\mathrm{c}-\mathrm{h}}$ & $0.41 \mathrm{hij}$ & $1.32^{\mathrm{c}}$ & $0.76 \mathrm{fg}$ & $0.78 \mathrm{abc}$ & $0.49^{\mathrm{jk}}$ & $0.47 \mathrm{k}$ & $0.28^{\mathrm{jik}}$ & $1.23 \mathrm{bc}$ & $1.18 \mathrm{de}$ & $1.43^{\mathrm{C}}$ & $0.68 \mathrm{j}^{\mathrm{k}}$ & $0.78^{\mathrm{c}}$ & $0.56 \mathrm{hij}$ & $1.43^{\mathrm{cde}}$ & $0.87 \mathrm{gh}$ \\
\hline QY 1 & $0.97 \mathrm{de}$ & $0.89 \mathrm{e}$ & $0.88^{\mathrm{d}}$ & $0.65^{\mathrm{cd}}$ & $0.70^{\mathrm{c}-\mathrm{h}}$ & 0.47 cde & $1.06 \mathrm{gh}$ & $0.86 \mathrm{e}$ & $0.76^{b-e}$ & $0.64^{\mathrm{b}}$ & $0.66^{\mathrm{hi}}$ & $0.35 \mathrm{fg}$ & $1.16^{\operatorname{def}}$ & $1.32 \mathrm{bc}$ & $1.41^{\mathrm{C}}$ & $0.69 \mathrm{jk}$ & $0.78^{\mathrm{c}}$ & $0.73 \mathrm{bc}$ & $\begin{array}{l}.1 .58 \mathrm{hi} \\
1.17 \mathrm{~h}\end{array}$ & $1.03 \mathrm{de}$ \\
\hline TYZ 283 & $1.01^{\mathrm{a}-\mathrm{d}}$ & $0.85^{\mathrm{h}}$ & $0.92 \mathrm{~cd}$ & 0.62 def & $0.75^{\mathrm{d}-\mathrm{h}}$ & $0.40^{\mathrm{ij}}$ & $1.12^{\mathrm{e}-\mathrm{h}}$ & $0.56^{\mathrm{i}}$ & $0.83^{\mathrm{a}}$ & $0.59 \mathrm{~cd}$ & $0.59 \mathrm{j}$ & $0.22^{1}$ & $1.18 \mathrm{bcd}$ & $1.17^{\mathrm{e}}$ & $1.20^{d}$ & $0.47 \mathrm{mn}$ & $0.84^{\mathrm{b}}$ & $0.60 \mathrm{fgh}$ & $1.17 \mathrm{hi}$ & $0.67 \mathrm{j}$ \\
\hline GZ1 & $1.00^{a-d}$ & $0.95 \mathrm{bc}$ & $0.82 \mathrm{e}$ & $0.59 \mathrm{fgh}$ & $0.76 \mathrm{bc}$ & $0.46 \mathrm{def}$ & $1.19 \mathrm{de}$ & $0.87 \mathrm{de}$ & $0.74^{\mathrm{c}-\mathrm{f}}$ & 0.56 def & $0.80 \mathrm{~d}$ & $0.31^{g-j}$ & $1.31 \mathrm{a}$ & $1.34^{\mathrm{a}}$ & $1.30^{\circ} \mathrm{C}$ & 0.70 ghi & $0.76 \mathrm{~cd}$ & $0.52 \mathrm{j}$ & $1.32 \mathrm{efg}$ & $1.01 \mathrm{e}^{\mathrm{e}}$ \\
\hline FY 520 & $1.011^{\mathrm{a}-\mathrm{d}}$ & $0.99 \mathrm{ab}$ & 0.78 efg & $0.68^{c}$ & $0.70^{\mathrm{e}-\mathrm{h}}$ & $0.48 \mathrm{bcd}$ & $0.85^{\mathrm{i}}$ & $0.69 \mathrm{gh}$ & $0.71 \mathrm{e}^{\mathrm{e}-\mathrm{i}}$ & $0.54 \mathrm{e}-\mathrm{h}$ & $0.64^{\mathrm{ij}}$ & $0.51^{\mathrm{cd}}$ & 1.06 fgh & $1.16 \mathrm{e}$ & $0.94 \mathrm{fg}$ & $0.74 \mathrm{fg}$ & 0.67 ghi & 0.56 hij & $0.88 \mathrm{j}$ & $0.78 \mathrm{ij}$ \\
\hline Mean & 0.99 & 0.87 & 0.84 & 0.58 & 0.72 & 0.42 & 1.16 & 0.76 & 0.71 & 0.53 & 0.76 & 0.38 & 1.10 & 1.10 & 1.15 & 0.68 & 0.75 & 0.60 & 1.34 & 0.99 \\
\hline
\end{tabular}

FG\%: final germination percentage; GR: germination rate; VI (I): vigor index (I); VI (II): vigor index (II); ShFW: shoot fresh weight; RFW: root fresh weight; ShDW: shoot dry weight RDW: root dry weight; ShL: shoot length, and RL: root length. Data are presented as mean values with different letters, which denote statistically significant difference between means within each indicator column among cultivars according to Fisher's least significant difference (LSD) test. 


\subsection{Correlations of Traits under Control and PEG-6000 Stress}

Pearson's correlations between the cultivars under normal and stressed conditions showed differences in response to drought stress. Correlations (r-value) of the 10 studied traits under 0, 10 and 15\% PEG-6000 treatments are presented in Figure 3A-C. Stronger correlations can be seen among traits, where r-values $\geq 0.7$ showed highly positively stronger relationship and $r$-values $\geq 0.5$ showed positively strong interaction. Under the nonstressed conditions, highly positive $r$ values $\geq 0.70$ were recorded for ShFW (0.73), RDW (0.81) and VI (I) (0.95) with ShL, RFW and RL, respectively. RFW was highly correlated with VI (I) and RL. Additionally, positive $r$ values $\geq 0.50$ were scored for ShDW with ShFW, and VI (II) with RDW, RFW and VI (I). FG\% showed a positive correlation with GR and VI (II), and GR with VI (II) and VI (I) (Figure 3A).
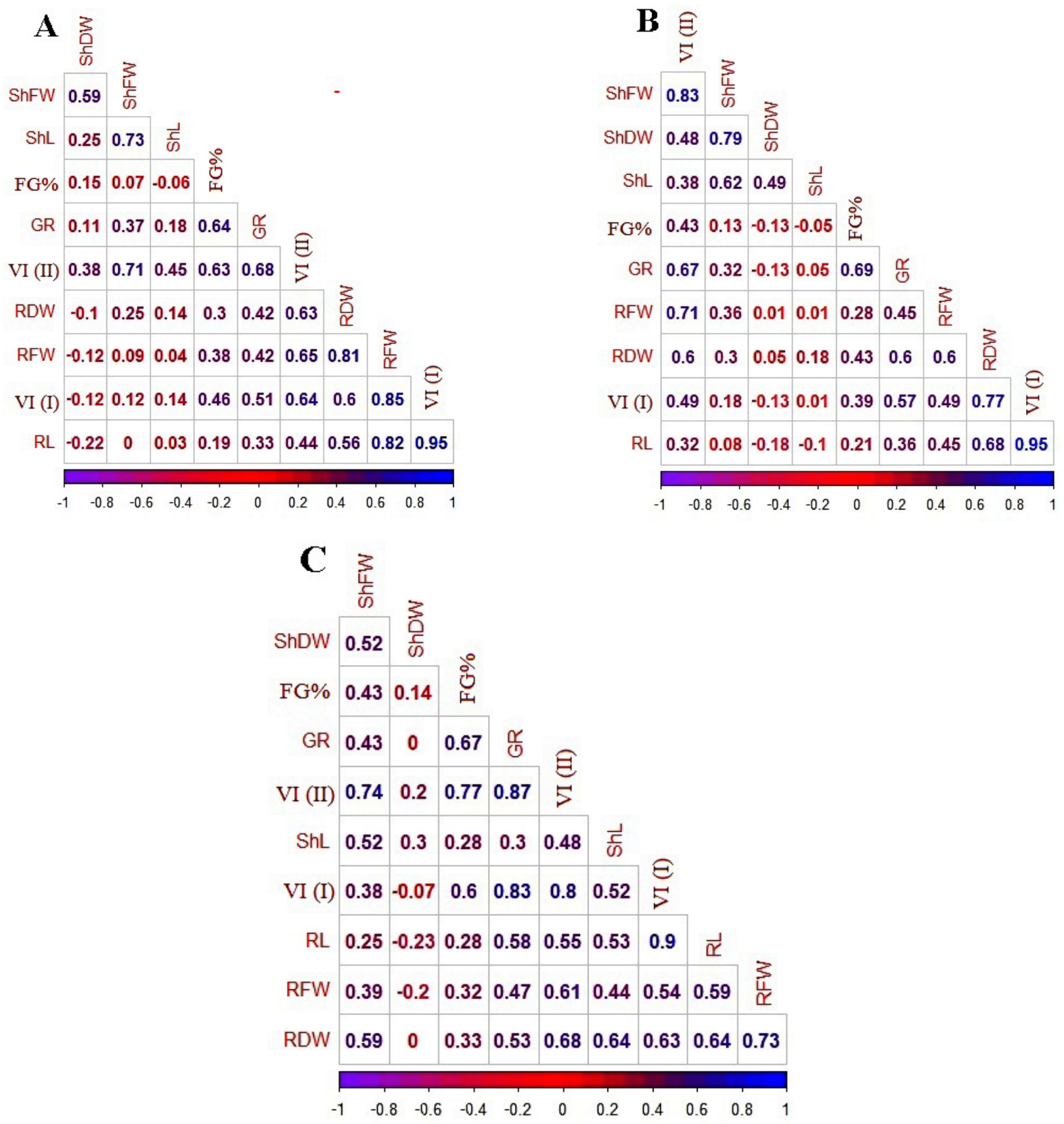

Figure 3. Correlation indicators of rapeseed cultivars based on mean values: under (A) control, (B) $10 \%$ PEG-6000 and (C) 15\% PEG-6000. 
Positive correlations were also observed for most of the same 10 traits under the $10 \%$ PEG-6000 treatment, as indicated by the red and yellow cells in the correlation triangle. The $r$ values were a little higher for some traits compared to their corresponding values under normal condition. Highly positive r-values were $\geq 0.70$ for VI (II) (0.83), ShFW (0.79), RDW (0.77) and VI (I) (0.95) seedlings with ShFW, ShDW, VI (I) and RL, respectively, indicating stronger correlation. Additionally, positive $\mathrm{r}$ values $\geq 0.50$ were scored for $\mathrm{FG} \%$, RFW and RDW with GR, RDW and RL, respectively. Lower values were observed for ShL, ShDW with all traits, except with ShL (Figure 3B). Furthermore, VI (II) was highly correlated with ShFW and FG\% with GR compared to control.

Under the 15\% PEG-6000 treatment, highly positive $r$ values were $\geq 0.70$ for VI (I) (0.90) and RFW (0.73) with RL and RDW, respectively. FG\% was correlated with VI (II) (0.77), while GR (r-value 0.83 and 0.87 ) was correlated with VI (I) and VI (II), with a stronger correlation. Meanwhile, positive $r$ values $\geq 0.50$ were recorded for ShFW with ShDW and ShL; RL with RFW and RDW, while lower values were obtained for ShDW with all attributes (Figure 3C).

Detailed inspection of the morphological traits showed that JYZ 158 and FY 520 cultivars had the best performance in several traits, while YG 2009 and NZ 1838 showed poor performance. According to the results, four cultivars were selected as sensitive and tolerant based on differences in drought tolerance and were further investigated with more measurements.

\subsection{Variation in Growth-Related Traits of Rapeseed Seedlings}

Based on germination and morphological traits analysis of 24 rapeseed cultivars, JYZ 158 and FY 520 were classed as highly tolerant cultivars, and YG 2009 and NZ 1838 classed as least tolerant cultivars. Results showed a significant reduction of the shoot and root length of rapeseed under drought stress, which was more prominent in sensitive cultivars (Figure 4). Highly tolerant cultivars (JYZ 158 and FY 520) and highly sensitive cultivars (YG 2009 and NZ) 1838 were selected to explore the role of osmolytes and antioxidant enzyme activity in improving drought tolerance.

V5 (JYZ 158)

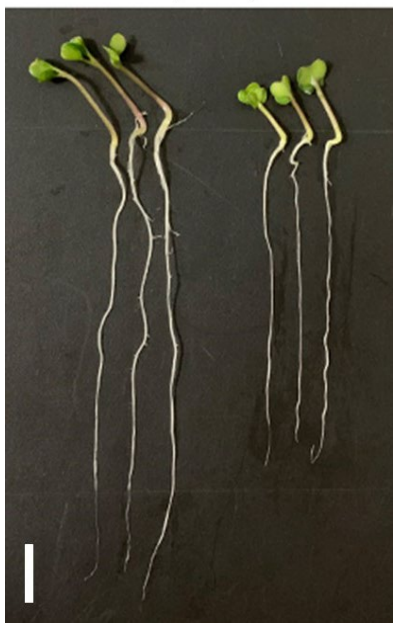

V24 (FY520)

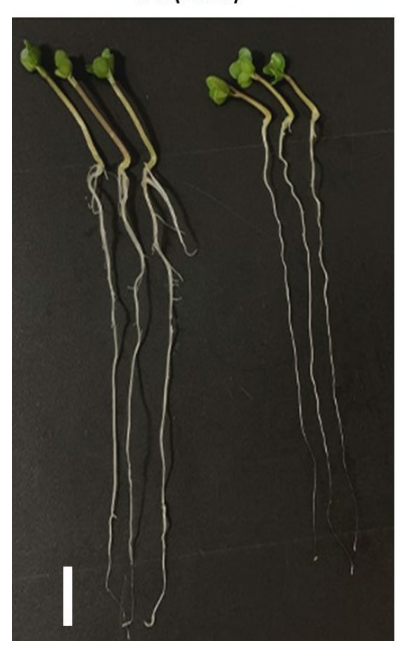

V19 (YG2009)

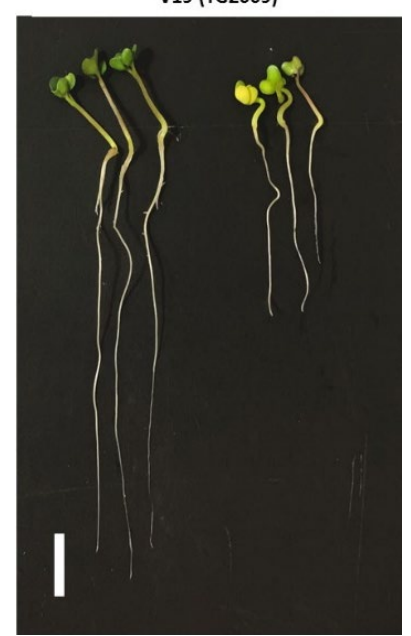

V12 (NZ1838)

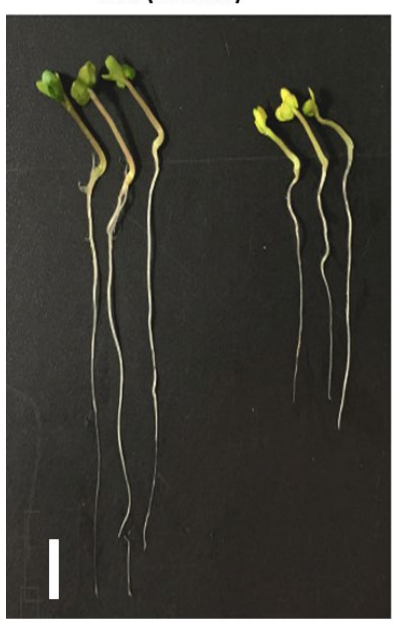

Figure 4. Effect of drought stress (15\% PEG-6000) on the shoot and root growth of tolerant cultivars (JYZ 158 and FY 520) and sensitive cultivars (YG 2009, and NZ 1838). Scale bar: $1 \mathrm{~cm}$.

\subsection{Variations in Photosynthetic Pigments under Drought Stress}

Compared to seedling growth under normal conditions, a significant decrease was noted in photosynthetic pigment levels under drought stress. Under 15\% PEG-6000, Chl a content was reduced by 21.22 and $23.21 \%$ in JYZ 158 and FY 520 and decreased by 27.32 and 26.61\% in YG 2009 and NZ 1838, respectively, while Chl b was decreased by 24.11\% (JYZ 158), 31.30\% (FY 520), 47.11\% (YG 2009) and 45.40\% (NZ 1838) (Figure 5A,B). Under 
stress, total chlorophyll in JYZ 158 and FY 520 (tolerant cultivars) was decreased by 21.93 and $25.21 \%$, while in YG 2009 and NZ 1838 (sensitive cultivars) it was reduced by 32.00 and $31.22 \%$, respectively (Figure 5 C). Carotenoid content was reduced by $24.90 \%$ (JYZ 158), $24.80 \%$ (FY 520), 30.16\% (YG 2009) and 29.02\% (NZ 1838) under 15\% PEG-6000 (Figure 5D).

A

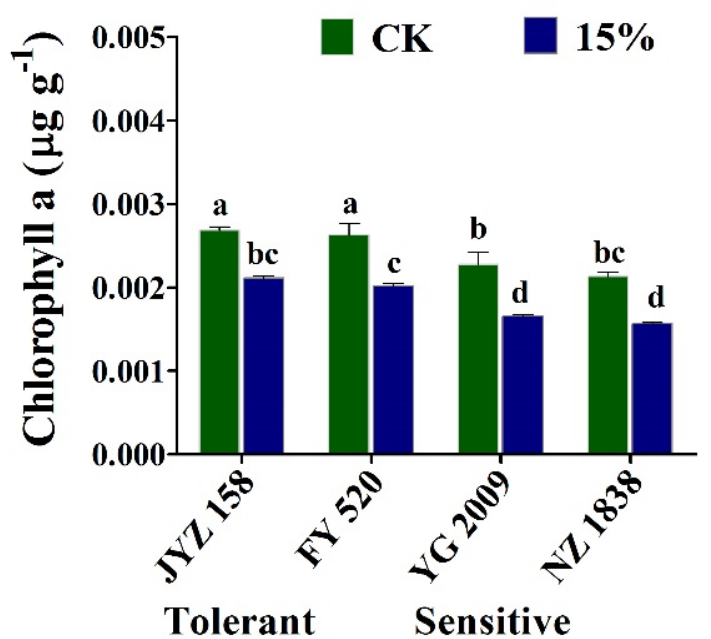

C

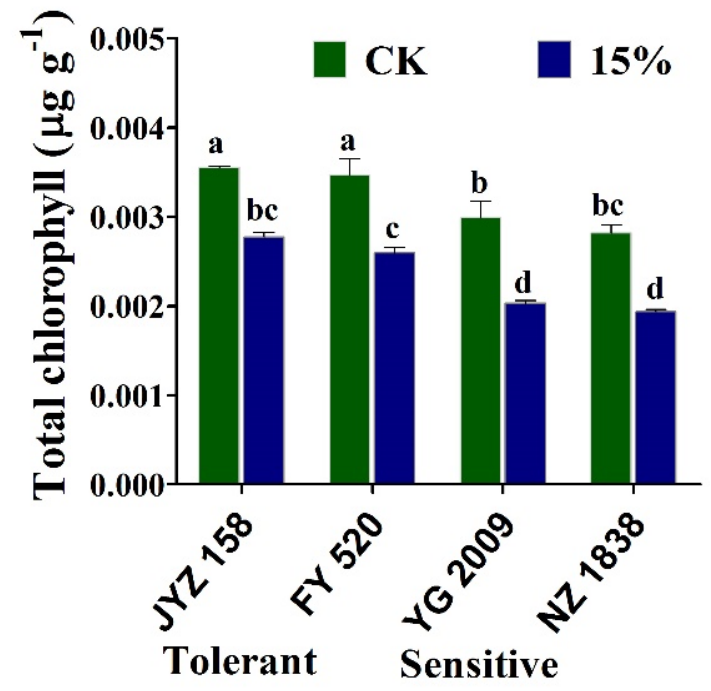

B

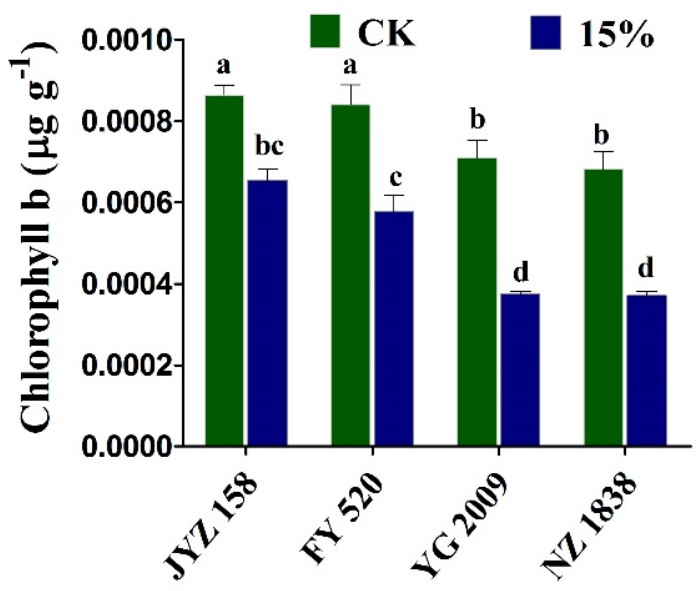

Tolerant Sensitive

D

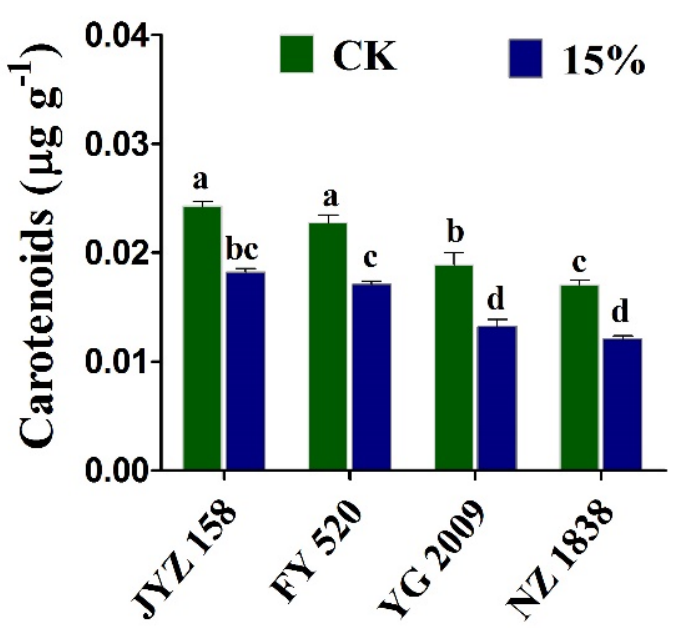

Tolerant Sensitive

Figure 5. Effects of drought stress (PEG-6000-induced) on (A) chlorophyll a, (B) chlorophyll b, (C) total chlorophyll and (D) carotenoids in four rapeseed cultivars during the early seedling stage. Bars represent mean $\pm \mathrm{SE}$ of three replicates. The different letters indicate significant differences at $p<0.05$ using Duncan's multiple range tests.

\subsection{Variation of Osmo-Protectants, MDA, Proline and RWC Contents in Rapeseed Seedlings}

Tolerant cultivars had higher levels of TSS and TSP than sensitive cultivars. Furthermore, TSS increased by 57.50, 45.83, 48.42 and 45.51\% in JYZ 158, FY 520, YG 2009 and NZ 1838 under drought stress, respectively, versus normal conditions. TSP was increased in JYZ 158 and FY 520 (tolerant cultivars), and YG 2009 and NZ 1838 (sensitive cultivars) by 14.81, $16.22,26.72$ and $24.21 \%$, respectively, over controls in relation to drought (Figure 6A,B). 
A

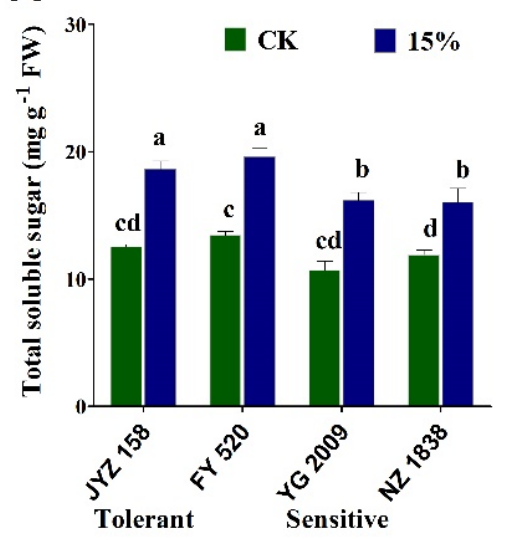

D

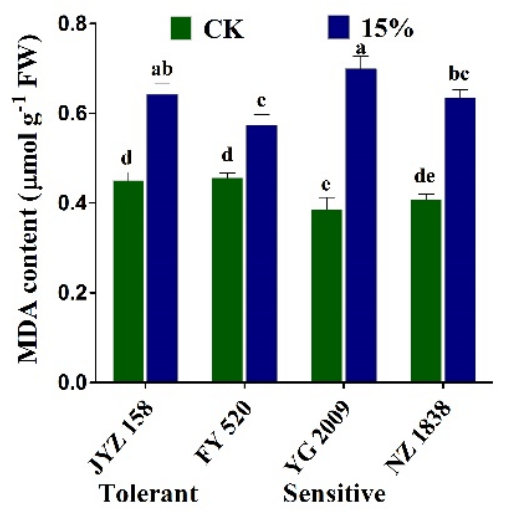

B

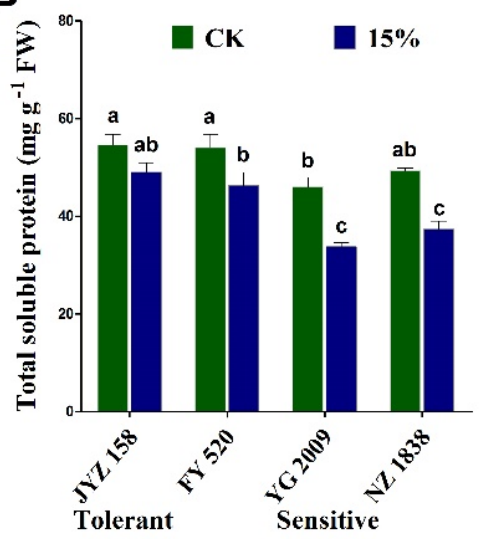

E

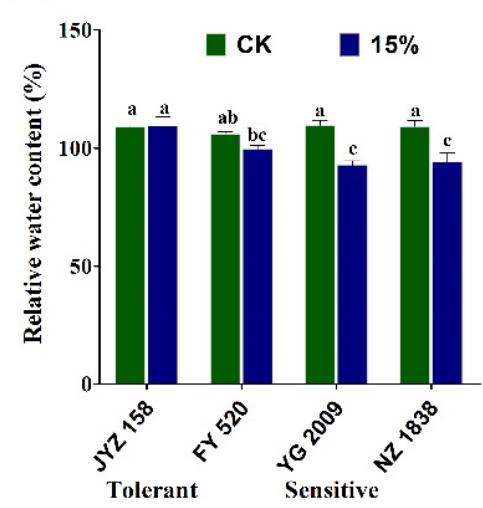

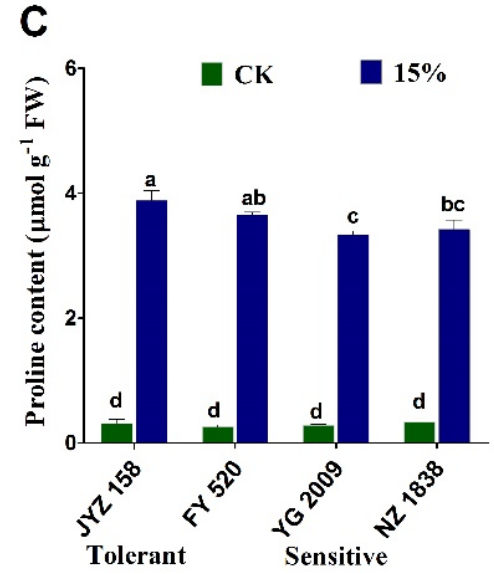

Figure 6. Effects of drought stress (PEG-6000-induced) on (A) total soluble sugar (TSS), (B) total soluble protein (TSP), (C) proline content, (D) MDA content and (E) water content in four rapeseed cultivars during the early seedling stage. Bars represent mean $\pm \mathrm{SE}$ of three replicates. Different letters indicate significant differences at $p<0.05$ using Duncan's multiple range tests.

The contents of proline and MDA under stress conditions were recorded as increases of 1161 and 42.72\% (JYZ 158), 1282 and 25.63\% (FY 520), 1072 and 81.51\% (YG 2009), 922.9 and $55.81 \%$ (NZ 1838), respectively, versus the normal condition (Figure 6C,D). RWC was stable in the tolerant cultivars under drought stress conditions compared to control; however, turgor was reduced significantly in sensitive cultivars due to weak tolerance. Water content was slightly enhanced by $0.42 \%$ in JYZ 158, slightly reduced by $6.101 \%$ in FY 520, and significantly reduced by 15.61 and 13.85\% in YG 2009 and NZ 1838, respectively, compared to control (Figure 6E).

\subsection{Activities of Enzymatic Antioxidants under PEG-6000 Induced Drought Stress}

Enzymatic antioxidants (Superoxide dismutase (SOD), peroxidase (POD), catalase (CAT) and ascorbate peroxidase (APX)) showed marked changes under drought stress in rapeseed seedlings of tolerant and sensitive cultivars. For SOD, JYZ 158 and FY 520 showed an increase of 116.3 and $167.4 \%$, respectively, while YG 2009 and NZ 1838 showed an increase of 85.62 and $71.31 \%$, respectively, under drought stress compared to control. POD activity increased under drought stress in all cultivars, by 89.92 and $69.50 \%$ in tolerant cultivars (JYZ 158 and FY 520), 96.91 and 79.23\% in sensitive cultivars (YG 2009 and NZ 1838) compared to control, indicating that POD activity might be a significant participant in the defense system (Figure 7A,B). CAT activity increased under drought stress by $108.8 \%$ (JYZ 158), 107.9\% (FY 520), 92.92\% (YG 2009), and 97.51\% (NZ 1838) compared to control, whereas APX activity increased by 143.5, 151.6, 34.31 and 212.1\% in JYZ 158, FY 520, YG 2009 and NZ 1838, respectively, under drought stress compared to control (Figure 7C,D). 
A

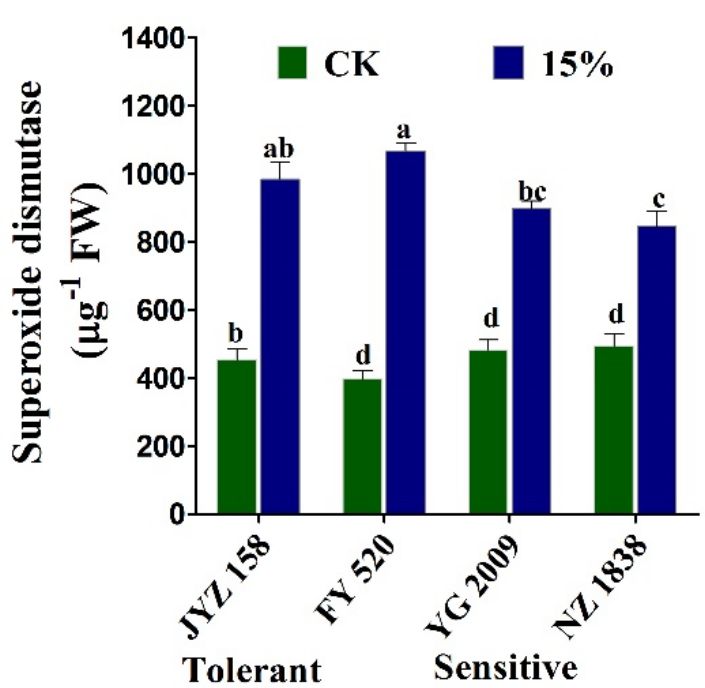

C

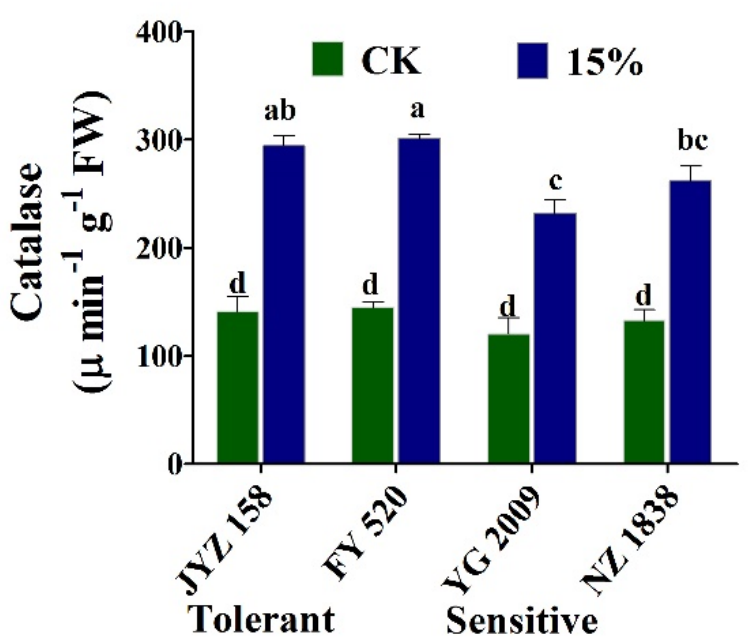

B

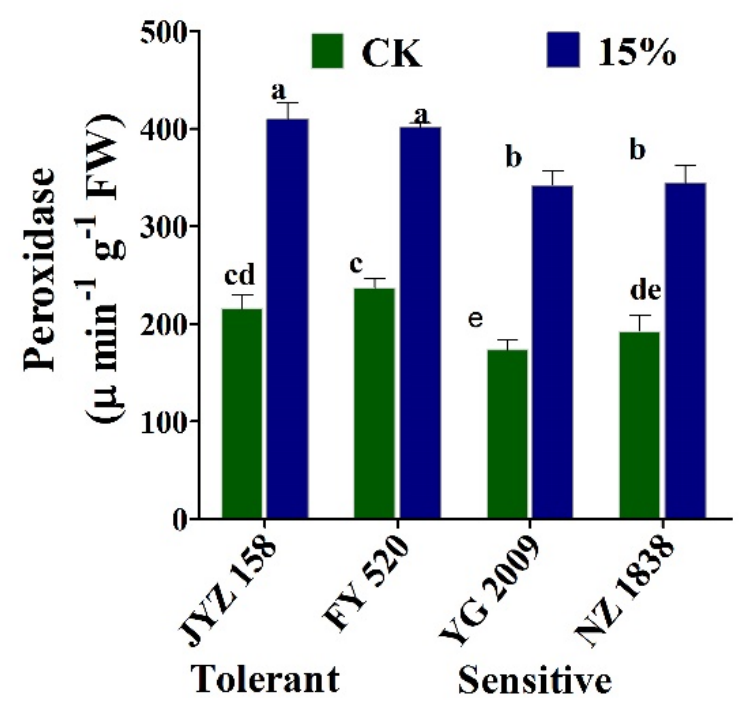

D

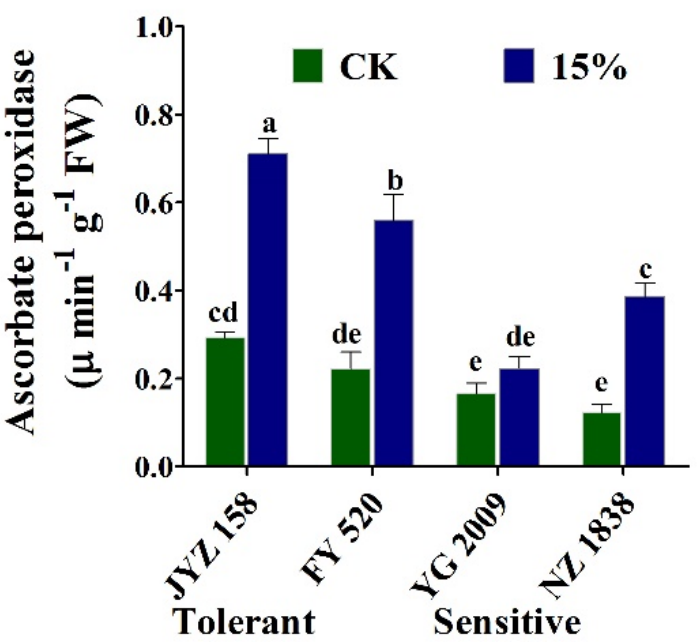

Figure 7. Effects of drought stress (PEG-6000 induced) on (A) superoxide dismutase (SOD), (B) peroxidase (POD), (C) catalase (CAT) and (D) ascorbate peroxidase (APX) in tolerant and sensitive rapeseed cultivars. Bars represent mean $\pm \mathrm{SE}$ of three replicates. The different letters indicate significant differences at $p<0.05$ using Duncan's multiple range tests.

\subsection{Microstructural Variation in Rapeseed Seedlings under Drought Stress}

To further investigate the effects of drought stress on the chloroplast, the ultrastructure of the chloroplast in two cultivars, FY 520 (highly tolerant) and YG 2009 (highly sensitive), was observed using transmission electron microscopy (TEM). Under normal conditions, the form of chloroplast was well organized with elliptic with clear edges and was positioned near the properly developed cell wall (Figure 8A,B). Under drought stress, the shape of chloroplasts was as well-organized as in the tolerant cultivars, with well-developed lamella having normally stacked grana and thylakoids, and the cell wall had a proper configuration (Figure 8C). However, the structure of chloroplasts in leaves of sensitive cultivars was abnormal, with several vesicles instead of thylakoids, and chloroplasts moved toward the 
center of the cell. Non-visible and incomplete cell boundaries were observed in sensitive cultivars under drought treatment (Figure 8D).
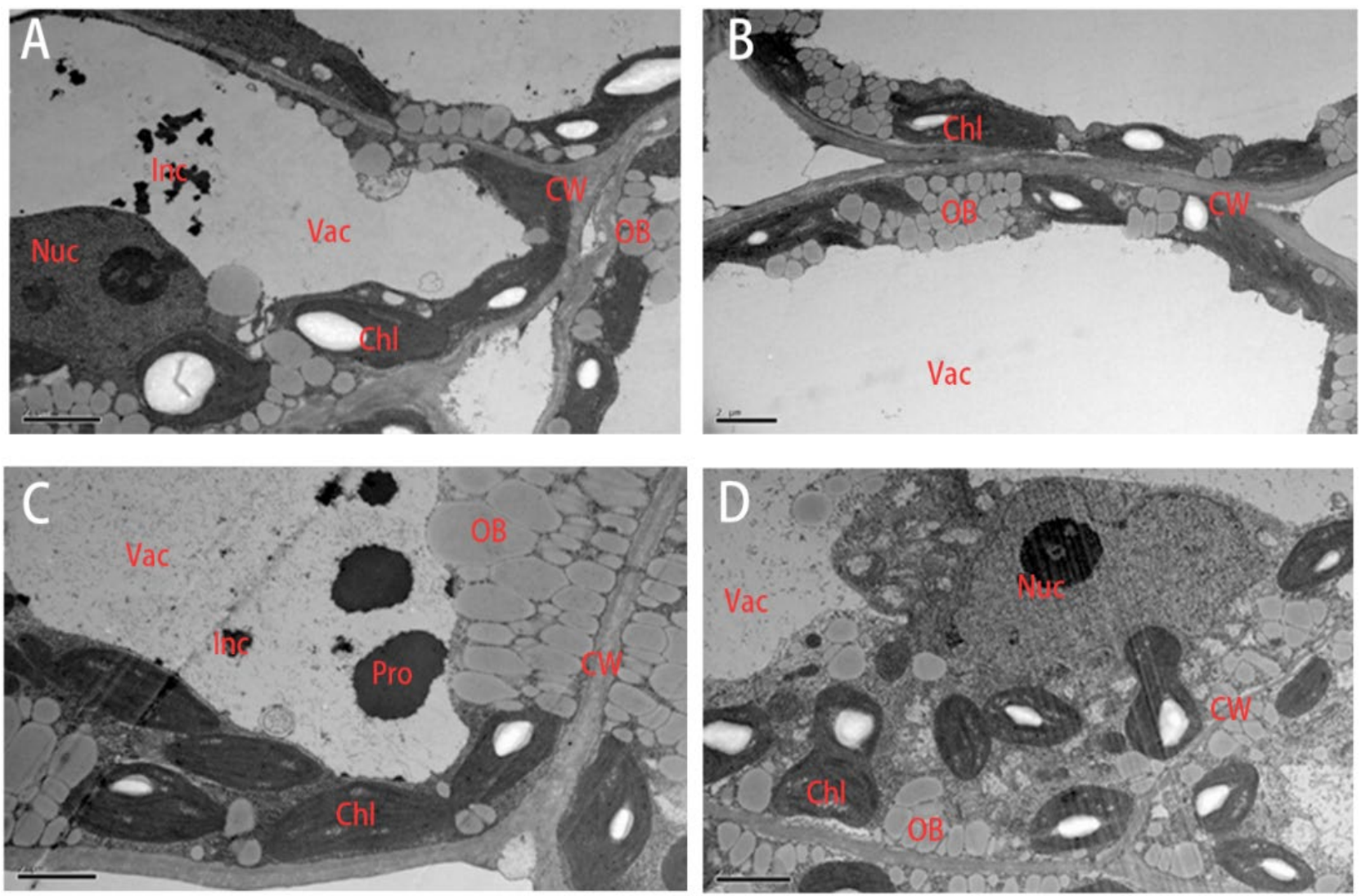

Figure 8. Effects of drought stress (PEG-6000-induced) on the internal structure of seedlings. (A) control of tolerant variety, (B) control of sensitive variety, (C) tolerant variety under 15\% PEG-6000 treatment and (D) sensitive variety with 15\% PEG-6000 treatment.

\section{Discussion}

Germination is a key step of seedling development during the plant life cycle [3]. A non-conducive environment, such as water stress, contributes towards poor seed germination and inhibits seedling development [35]. Rapid germination and successful seedling establishment are crucial for the normal growth and profitable production of Brassica napus L. $[36,37]$. The current study showed that germination percentage, germination rate and seedling growth were considerably decreased in all 24 studied cultivars under PEG-6000-simulated drought stress, but the negative effect was higher in sensitive cultivars compared to tolerant cultivars. Hence, germination percentage and germination speed was reduced under stress condition, which would lead to poor stand establishment [3,38]. Reduced germination was due to reduced water uptake, lower energy supply and impairment of enzymatic activities [39].

Drought stress reduces the water potential gradient between the internal and external environment of seeds [40] and reduces water movement through the seed coat and water absorption [41], resulting in reduction and delayed seed germination [3]. Drought stress reduced germination and seedling growth in B. napus and enhanced ROS production, which has damaging effects on structural components of cells and metabolic processes [11]. The slower hydrolysis of materials present in the endosperm leads to a lower transportation rate of hydrolyzed material to the developing embryonic axis, reducing germination and growth [11,42].

The current investigation showed that the shoot length of seedlings was reduced under drought stress due to a reduction in water availability [43]. Moreover, a substantial 
reduction in plant height, leaf size and chlorophyll content occurred under water deficiency in rapeseed [44]. Our results show that mild drought stress increased root length, which indicates that mild water deficit might cause alterations in root structure to prevent dehydration [45]. On the other hand, severe drought stress shortened root length and reduced development in the 24 studied cultivars, indicating that a significant reduction in root length was due to reduction in cell division and expansion [46]. Drought stress causes disturbance of several physiochemical process, with a complex mechanistic action that limits plant development [47-50]. A few cultivars maintained or had greater germination and seedling growth under 10\% PEG-6000, suggesting that plants possess an effective defense system that may be stimulated with higher efficacy under moderate stress, ultimately enabling the plants to grow better under drought stress conditions [51].

Photosynthetic pigments decreased in the four studied cultivars, but the reduction was less in drought-tolerant than sensitive cultivars, indicating that photoinhibition of photosystem II was higher [52]. A decline in chlorophyll content is usually observed during drought exposure, and it causes a significant reduction in carotenoids and chlorophyll biosynthesis [53]. Drought stress causes dysfunction/destruction of the thylakoid structural membrane, which leads to a drastic decrease in chlorophyll content under water stress [54]. Additionally, drought affects chlorophyll-based chiral macro-aggregates of the harvesting complex, which cause oxidative stress [55]. The level of $\mathrm{Chl}$ a and b were significantly higher for irrigated plants than water-stressed plants in rapeseed [56], which supports our results that seedlings under normal conditions had significantly higher chlorophyll contents than drought treated seedlings. A detrimental effect of water deficiency was degeneration of chlorophyll, which causes a decline in the energy transfer between chlorophyll and the reaction center [54] and induces the overproduction of electrons through the electron transport chain, damaging the photosynthetic apparatus [57].

The present study showed that drought stress greatly influenced synthesis of the plant cell wall. The structural integrity of cells in the leaves of sensitive cultivars was greatly affected and abnormally formed compared to the tolerant cultivar, with oval to round chloroplasts moving toward the center of the cell. Furthermore, nonvisible and incomplete boundaries of the cell were observed under drought stress. These results coincide with those of maize seedlings, where the chloroplast structure varied from oval to circular due to plasmolysis caused by drought stress [17]. Additionally, chloroplast degeneration showed variation between tolerant and sensitive cultivars under drought stress [58-60].

The plant is a sessile organism and its responds to an unfavorable environment, such as drought, by a signaling pathway resulting in adaptation [61]. Water shortage causes oxidative stress in tissues and induces electron leakage within mitochondria and chloroplasts that leads to excitation of triplet oxygen, and enhanced ROS. This disorganizes the structure of photosynthetic pigments, consequently reducing photosynthesis and biomass production [62].

Substantial damage by ROS was recorded, which favor lipid peroxidation and structural degradation in stressed plants [22]. Osmotic adjustment and compatible solutes play a key role against drought stress by stabilizing cellular structure and function and maintaining turgor [63]. Accumulated solutes of different lower molecular weights, including TSS, proline, glycine betaine (GB), organic acids (OA) and trehalose, protect cell structure, thereby maintaining functional activity $[64,65]$. Proline is an important metabolite that accumulates under drought stress and confers protection on the sub-cellular structure and increases the activity of anti-oxidants [64], leading to an appreciable increase in drought tolerance. TSS and proline levels increased under drought stress in the four studied cultivars, signifying the role of osmolyte in all cultivars under drought stress, and indicating that several metabolites accumulated to relieve osmotic stress [66], and enhance plant survival.

Drought tolerance is correlated with an efficient scavenging system that helps maintain low ROS, thus preventing membrane peroxidation [9]. The response mechanism in plants against drought conditions depends on antioxidative enzymatic activity and osmolytes accumulation. CAT, SOD, POD and APX are essential enzymes in the defensive mechanism 
that scavenges ROS. For the destruction of $\mathrm{H}_{2} \mathrm{O}_{2}$, several antioxidative enzymes act in synchrony. SOD is involved in the conversion of $\mathrm{O}_{2}{ }^{-}$into $\mathrm{H}_{2} \mathrm{O}_{2}$ and $\mathrm{O}_{2}$, while CAT and POD convert $\mathrm{H}_{2} \mathrm{O}_{2}$ into $\mathrm{O}_{2}$ and $\mathrm{H}_{2} \mathrm{O}$, and APX is involved in the AsA-GSH cycle, that supports the $\mathrm{H}_{2} \mathrm{O}_{2}$ removal [24]. This study demonstrated that such enzymatic activities increased under drought stress in the four studied cultivars, and the enhancement was higher in tolerant than in sensitive cultivars, suggesting that tolerant cultivars have a more efficient defense system, including enhanced scavenging activity [67].

\section{Conclusions}

Germination and growth-related traits showed variation among all studied cultivars, showing that tolerance against drought stress varied with exposure level and cultivar. The results show that drought negatively affects seed germination and seedling growth. Rapeseed seedlings respond to stress conditions with adaptive and acclimatization strategies, ranging from seemingly simple morphological responses to complex physiochemical changes that serve as important stress tolerance markers. The tolerance capacity was different for different cultivars. JYZ 158 and FY 520 had greater drought tolerance, while YG 2009 and NZ 1838 had lower drought tolerance. The study showed that drought stress imparted negative impacts on development and induced defense mechanisms for protection against drought-induced injuries. Drought tolerance in tolerant cultivars was due to higher antioxidant activity through enzymes and osmotic adjustment by accumulating osmotic substances such as proline, total soluble sugar and protein. Our findings provide insight into the drought-responsive mechanisms that can assist the researchers in improving the tolerance of rapeseed cultivars. The outcomes of this investigation have important implications for research on rapeseed during seed germination and at the early seedling stage during drought stress.

Supplementary Materials: The following supporting information can be downloaded at: https: / / www.mdpi.com/article/10.3390/agronomy12030579/s1. Table S1: List of 24 cultivars examined under PEG-6000-induced drought stress. Table S2: Germination and seedling traits description and abbreviations under control and polyethylene glycol 6000 (PEG-6000) drought treatments, and the drought tolerance indices (DTIs) used to evaluate traits response to drought treatments. Table S3: Final germination percentage of three cultivars to select the concentration of PEG-6000.

Author Contributions: Conceptualization, M.B. and A.M.E.-B.; methodology, M.B.; software, I.A.A.M. and H.Y.; formal analysis, Z.W., X.A. and A.S.; investigation, B.W. and J.K.; resources, B.W. and G.Z.; data curation, R.S.; validation, M.U.H.; writing-original draft preparation, M.B. and A.M.E.-B.; writing-review \& editing, M.B. and A.M.E.-B.; supervision, B.W.; project administration, B.W.; funding acquisition, B.W. and G.Z. All authors have read and agreed to the published version of the manuscript.

Funding: This research was funded by "National Key Research and Development Program of China, 2020 YFD1000904" and "Technical Innovation Project in Hubei Province, 2020 BBB061, and 2020 BBB062".

Institutional Review Board Statement: Not applicable.

Informed Consent Statement: Not applicable.

Data Availability Statement: Not applicable.

Acknowledgments: The authors are thankful to all lab mates for their support.

Conflicts of Interest: The authors declare no conflict of interest.

\section{References}

1. Yan, L.; Shah, T.; Cheng, Y.; LÜ, Y.; Zhang, X.K.; Zou, X.L. Physiological and molecular responses to cold stress in rapeseed (Brassica napus L.). J. Integr. Agric. 2019, 18, 2742-2752. [CrossRef]

2. $\quad \mathrm{Wu}, \mathrm{W} . ; \mathrm{Ma}$, B.L.L.; Whalen, J.K. Enhancing Rapeseed Tolerance to Heat and Drought Stresses in a Changing Climate: Perspectives for Stress Adaptation from Root System Architecture. Adv. Agron. 2018, 151, 87-157. 
3. Channaoui, S.; Kahkahi, R.E.; Charafi, J.; Mazouz, H.; Fechtali, M.E.; Nabloussi, A. Germination and Seedling Growth of a Set of Rapeseed (Brassica napus) Varieties under Drought Stress Conditions. Int. J. Environ. Agric. Biotechnol. 2017, 2, 487-494. [CrossRef]

4. Zhu, M.; Monroe, J.G.; Suhail, Y.; Villiers, F.; Mullen, J.; Pater, D.; Hauser, F.; Jeon, B.W.; Bader, J.S.; Kwak, J.M.; et al. Molecular and systems approaches towards drought-tolerant canola crops. New Phytol. 2016, 210, 1169-1189. [CrossRef] [PubMed]

5. Tandzi, L.N.; Bradley, G.; Mutengwa, C. Morphological Responses of Maize to Drought, Heat and Combined Stresses at Seedling Stage. J. Biol. Sci. 2018, 19, 7-16. [CrossRef]

6. Yan, C.; Song, S.; Wang, W.; Wang, C.; Li, H.; Wang, F.; Li, S.; Sun, X. Screening diverse soybean genotypes for drought tolerance by membership function value based on multiple traits and drought-tolerant coefficient of yield. BMC Plant Biol. 2020, 20, 321. [CrossRef]

7. Dani, A.R.H.; Siswoyo, T.A. Impact of Drought Stress during Germination on Antioxidant Capacities and Antioxidant Enzymes Activities of Madura Local Maize (Zea mays) Seeds. Agric. Sci. 2019, 10, 1506-1516. [CrossRef]

8. Razaji, A.; Farzanian, M.; Sayfzadeh, S. The effects of seed priming by ascorbic acid on some morphological and biochemical aspects of rapeseed (Brassica napus L.) under drought stress condition. Int. J. Biosci. 2014, 4, 432-442.

9. Hasanuzzaman, M.; Nahar, K.; Anee, T.I.; Khan, M.I.R.; Fujita, M. Silicon-mediated regulation of antioxidant defense and glyoxalase systems confers drought stress tolerance in Brassica napus L. S. Afr. J. Bot. 2018, 115, 50-57. [CrossRef]

10. El-Badri, A.M.; Batool, M.; AA Mohamed, I.; Wang, Z.; Khatab, A.; Sherif, A.; Ahmad, H.; Khan, M.N.; Hassan, H.M.; Elrewainy, I.M. Antioxidative and Metabolic Contribution to Salinity Stress Responses in Two Rapeseed Cultivars during the Early Seedling Stage. Antioxidants 2021, 10, 1227. [CrossRef]

11. Khan, M.N.; Zhang, J.; Luo, T.; Liu, J.; Ni, F.; Rizwan, M.; Fahad, S.; Hu, L. Morpho-physiological and biochemical responses of tolerant and sensitive rapeseed cultivars to drought stress during early seedling growth stage. Acta Physiol. Plant. 2019, 41, 25. [CrossRef]

12. Basal, O.; Szabó, A.; Veres, S. PEG-induced drought stress effects on soybean germination parameters. J. Plant Nutr. 2020, 43, 1768-1779. [CrossRef]

13. Kaydan, D.; Yagmur, M. Germination, seedling growth and relative water content of shoot in different seed sizes of triticale under osmotic stress of water and NaCI. Afr. J. Biotechnol. 2008, 7, 2862-2868. [CrossRef]

14. Abdel-Ghani, A.H.; Neumann, K.; Wabila, C.; Sharma, R.; Dhanagond, S.; Owais, S.J.; Börner, A.; Graner, A.; Kilian, B. Diversity of germination and seedling traits in a spring barley (Hordeum vulgare L.) collection under drought simulated conditions. Genet. Resour. Crop Evol. 2015, 62, 275-292. [CrossRef]

15. Mamnabi, S.; Nasrollahzadeh, S.; Ghassemi-Golezani, K.; Raei, Y. Improving yield-related physiological characteristics of spring rapeseed by integrated fertilizer management under water deficit conditions. Saudi J. Biol. Sci. 2020, 27, 797-804. [CrossRef]

16. Hellal, F.A.; El-Shabrawi, H.M.; Abd El-Hady, M.; Khatab, I.A.; El-Sayed, S.A.A.; Abdelly, C. Influence of PEG induced drought stress on molecular and biochemical constituents and seedling growth of Egyptian barley cultivars. J. Genet. Eng. Biotechnol. 2018, 16, 203-212. [CrossRef] [PubMed]

17. Zhang, F.-J.; Zhang, K.-K.; Du, C.-Z.; Li, J.; Xing, Y.-X.; Yang, L.-T.; Li, Y.-R. Effect of drought stress on anatomical structure and chloroplast ultrastructure in leaves of sugarcane. Sugar Tech 2015, 17, 41-48. [CrossRef]

18. Cheng, L.; Han, M.; Yang, L.m.; Li, Y.; Sun, Z.; Zhang, T. Changes in the physiological characteristics and baicalin biosynthesis metabolism of Scutellaria baicalensis Georgi under drought stress. Ind. Crops Prod. 2018, 122, 473-482. [CrossRef]

19. Bhargava, S.; Sawant, K. Drought stress adaptation: Metabolic adjustment and regulation of gene expression. Plant Breed. 2013, 132, 21-32. [CrossRef]

20. Kocsy, G.; Tari, I.; Vanková, R.; Zechmann, B.; Gulyás, Z.; Poór, P.; Galiba, G. Redox control of plant growth and development. Plant Sci. 2013, 211, 77-91. [CrossRef]

21. Sohag, A.A.M.; Tahjib-Ul-Arif, M.; Brestič, M.; Afrin, S.; Sakil, M.A.; Hossain, M.T.; Hossain, M.A.; Hossain, M.A. Exogenous salicylic acid and hydrogen peroxide attenuate drought stress in rice. Plant Soil Environ. 2020, 66, 7-13. [CrossRef]

22. Xiao, S.; Liu, L.; Wang, H.; Li, D.; Bai, Z.; Zhang, Y.; Sun, H.; Zhang, K.; Li, C. Exogenous melatonin accelerates seed germination in cotton (Gossypium hirsutum L.). PLoS ONE 2018, 14, e0216575. [CrossRef] [PubMed]

23. Ahammed, G.J.; Wang, Y.; Mao, Q.; Wu, M.; Yan, Y.; Ren, J.; Wang, X.; Liu, A.; Chen, S. Dopamine alleviates bisphenol A-induced phytotoxicity by enhancing antioxidant and detoxification potential in cucumber. Environ. Pollut. 2020, 259, 113957. [CrossRef] [PubMed]

24. Gao, S.; Wang, Y.; Yu, S.; Huang, Y.; Liu, H.; Chen, W.; He, X. Effects of drought stress on growth, physiology and secondary metabolites of Two Adonis species in Northeast China. Sci. Hortic. 2020, 259, 108795. [CrossRef]

25. Kumari, N.; Yadav, M.; Sharma, V. Differential response of Brassica juncea cultivars to al; consequences for chlorophyll a fluorescence, antioxidants and psb a gene. J. Plant Interact. 2018, 13, 496-505. [CrossRef]

26. Ju, Y.-L.; Yue, X.-F.; Zhao, X.-F.; Zhao, H.; Fang, Y.-L. Physiological, micro-morphological and metabolomic analysis of grapevine (Vitis vinifera L.) leaf of plants under water stress. Plant Physiol. Biochem. 2018, 130, 501-510. [CrossRef]

27. Meneses, C.H.S.G.; Bruno, R.D.L.A.; Fernandes, P.D.; Pereira, W.E.; Lima, L.H.G.D.M.; Lima, M.M.D.A.; Vidal, M.S. Germination of cotton cultivar seeds under water stress induced by polyethyleneglycol-6000. Sci. Agric. 2011, 68, 131-138. [CrossRef]

28. Badr, A.; El-Shazly, H.H.; Tarawneh, R.A.; Börner, A. Screening for drought tolerance in maize (Zea mays L.) germplasm using germination and seedling traits under simulated drought conditions. Plants 2020, 9, 565. [CrossRef] 
29. El-Badri, A.M.; Batool, M.; Mohamed, I.A.; Khatab, A.; Sherif, A.; Wang, Z.K.; Salah, A.; Nishawy, E.; Ayaad, M.; Kuai, J. Modulation of salinity impact on early seedling stage via nano-priming application of Zinc oxide on rapeseed (Brassica napus L.). Plant Physiol. Biochem. 2021, 166, 376-392. [CrossRef]

30. Zhang, Z.J.; Li, H.Z.; Zhou, W.J.; Takeuchi, Y.; Yoneyama, K. Effect of 5-aminolevulinic acid on development and salt tolerance of potato (Solanum tuberosum L.) microtubers in vitro. Plant Growth Regul. 2006, 49, 27-34. [CrossRef]

31. Yasmeen, A.; Basra, S.M.A.; Wahid, A.; Nouman, W.; Rehman, H.U. Exploring the potential of Moringa oleifera leaf extract (MLE) as a seed priming agent in improving wheat performance. Turk. J. Bot. 2013, 37, 512-520. [CrossRef]

32. Subramanyam, K.; Laing, G.D.; Van Damme, E.J.M. Sodium selenate treatment using a combination of seed priming and foliar spray alleviates salinity stress in rice. Front. Plant Sci. 2019, 10, 116. [CrossRef] [PubMed]

33. Heath, R.L.; Packer, L. Photoperoxidation in isolated chloroplasts. I. Kinetics and stoichiometry of fatty acid peroxidation. Arch Biochem. Biophys. 1968, 125, 189-198. [CrossRef]

34. Kong, Y.; Xu, X.; Zhu, L. Cyanobactericidal Effect of Streptomyces sp. HJC-D1 on Microcystis auruginosa. PLoS ONE 2013, 8, e57654. [CrossRef]

35. Hussain, H.A.; Hussain, S.; Khaliq, A.; Ashraf, U.; Anjum, S.A.; Men, S.; Wang, L. Chilling and drought stresses in crop plants: Implications, cross talk, and potential management opportunities. Front. Plant Sci. 2018, 9, 393. [CrossRef]

36. Jian, H.; Wang, J.; Wang, T.; Wei, L.; Li, J.; Liu, L. Identification of Rapeseed MicroRNAs Involved in Early Stage Seed Germination under Salt and Drought Stresses. Front. Plant Sci. 2016, 7, 7. [CrossRef]

37. Finch-Savage, W.E.; Clay, H.A.; Lynn, J.R.; Morris, K. Towards a genetic understanding of seed vigour in small-seeded crops using natural variation in Brassica oleracea. Plant Sci. 2010, 179, 582-589. [CrossRef]

38. Shahverdikandi, M.A.; Tobeh, A.; Godehkahriz, S.J.; Rastegar, Z. The study of germination index of canola cultivars for drought resistance. Int. J. Agron. Plant Prod 2011, 2, 89-95.

39. Okçu, G.; Kaya, M.D.; Atak, M. Effects of salt and drought stresses on germination and seedling growth of pea (Pisum sativum L.). Turk. J. Agric. For. 2005, 29, 237-242. [CrossRef]

40. Ajirloo, A.R.; Mohammadi, G.R.; Ghobadi, M. The Effect of Priming on Seed Performance of Chickpea (Cicer arietinum L.) under Drought Stress. J. Agric. Environ. Sci. 2011, 1, 1349-1351.

41. Boureima, S.; Eyletters, M.; Diouf, M.; Diop, T.A.; Damme, P.V. Sensitivity of Seed Germination and Seedling Radicle Growth to Drought Stress in Sesame (Sesamum indicum L.). Res. J. Environ. Sci. 2011, 5, 557-564. [CrossRef]

42. Ayaz, F.A.; Kadioglu, A.; Turgut, R. Water stress effects on the content of low molecular weight carbohydrates and phenolic acids in Ctenanthe setosa (Rosc.) Eichler. Can. J. Plant Sci. 2000, 80, 373-378. [CrossRef]

43. Li, J.; Zeng, L.; Cheng, Y.; Lu, G.; Fu, G.; Ma, H.; Liu, Q.; Zhang, X.; Zou, X.; Li, C. Exogenous melatonin alleviates damage from drought stress in Brassica napus L. (rapeseed) seedlings. Acta Physiol. Plant. 2018, 40, 43. [CrossRef]

44. Sharif, P.; Seyedsalehi, M.; Paladino, O.; Van Damme, P.; Sillanpää, M.; Sharifi, A.A. Effect of drought and salinity stresses on morphological and physiological characteristics of canola. Int. J. Environ. Sci. Technol. 2018, 15, 1859-1866. [CrossRef]

45. Hasibeder, R.; Fuchslueger, L.; Richter, A.; Bahn, M. Summer drought alters carbon allocation to roots and root respiration in mountain grassland. New Phytol. 2015, 205, 1117-1127. [CrossRef]

46. Ashraf, M.; Shahbaz, M.; Ali, Q. Drought-induced modulation in growth and mineral nutrients in canola (Brassica napus L.). Pak. J. Bot. 2013, 45, 93-98.

47. Umair Hassan, M.; Aamer, M.; Umer Chattha, M.; Haiying, T.; Shahzad, B.; Barbanti, L.; Nawaz, M.; Rasheed, A.; Afzal, A.; Liu, Y.; et al. The Critical Role of Zinc in Plants Facing the Drought Stress. Agriculture 2020, 10, 396. [CrossRef]

48. Batool, M.; El-Badri, A.M.; Hassan, M.U.; Haiyun, Y.; Chunyun, W.; Zhenkun, Y.; Jie, K.; Wang, B.; Zhou, G. Drought Stress in Brassica napus: Effects, Tolerance Mechanisms, and Management Strategies. J. Plant Growth Regul. 2022, 1-25. [CrossRef]

49. Hassan, M.; Aamer, M.; Chattha, M.; Ullah, M.; Suleman, S.; Nawaz, M.; Yanqin, M.; Huang, G. The role or potassium in plants under drought stress; Mini review. J. Basic Appl. Sci. 2017, 13, 268-271. [CrossRef]

50. Rasheed, A.; Hassan, M.U.; Aamer, M.; Batool, M.; Fang, S.; WU, Z.; LI, H. A Critical Review on the Improvement of Drought Stress Tolerance in Rice (Oryza sativa L.). Not. Bot. Horti Agrobot. Cluj-Napoca 2020, 48, 1756-1788. [CrossRef]

51. Jing, D.W.; Xing, S.J.; Du, Z.Y.; Liu, F.C. Effects of drought stress on the growth, photosynthetic characteristics, and active oxygen metabolism of poplar seedlings. Chin. J. Appl. Ecol. 2013, 24, 1809-1816.

52. Zhang, J.; Mason, A.S.; Wu, J.; Liu, S.; Zhang, X.; Luo, T.; Redden, R.; Batley, J.; Hu, L.; Yan, G. Identification of putative candidate genes for water stress tolerance in canola (Brassica napus). Front. Plant Sci. 2015, 6, 1058. [CrossRef] [PubMed]

53. Ragab, M.E.; Helal, N.A.S.; Sawan, O.M.; Fawzy, Z.F.; El Sawy, S.M. Foliar application of glycine betaine for alleviating water stress of tomato plants grown under sandy soil conditions. Int. J. ChemTech Res. 2015, 8, 52-67.

54. Chen, Y.E.; Liu, W.J.; Su, Y.Q.; Cui, J.M.; Zhang, Z.W.; Yuan, M.; Zhang, H.Y.; Yuan, S. Different response of photosystem II to short and long-term drought stress in Arabidopsis thaliana. Physiol. Plant. 2016, 158, 225-235. [CrossRef] [PubMed]

55. Guo, L.P.; Kang, H.J.; Ouyang, Z.; Zhuang, W.; Yu, Q. Photosynthetic parameter estimations by considering interactive effects of light, temperature and CO2 concentration. Int. J. Plant Prod. 2015, 9, 321-346. [CrossRef]

56. Zhang, X.; Lu, G.; Long, W.; Zou, X.; Li, F.; Nishio, T. Recent progress in drought and salt tolerance studies in Brassica crops. Breed. Sci. 2014, 64, 60-73. [CrossRef] [PubMed]

57. Gill, S.S.; Tuteja, N. Reactive oxygen species and antioxidant machinery in abiotic stress tolerance in crop plants. Plant Physiol. Biochem. 2010, 48, 909-930. [CrossRef] 
58. Bai, Z.; Li, C.; Qu, P. Effect of drought stress on ultrastructure of flag leaves in wheat chromosome substitution lines. J. Chin. Electron Microsc. Soc. 2009, 28, 68-73.

59. Chen, J.; Li, R.; Guo, P.; Xia, Y.; Tian, C.; Miao, S. Impact of drought stress on the ultrastructure of leaf cells in three barley genotypes differing in level of drought tolerance. Chin. Bull. Bot. 2011, 46, 28.

60. Zhang, Y.-B.; Yang, S.-L.; Dao, J.-M.; Deng, J.; Shahzad, A.N.; Fan, X.; Li, R.-D.; Quan, Y.-J.; Bukhari, S.A.H.; Zeng, Z.-H. Drought-induced alterations in photosynthetic, ultrastructural and biochemical traits of contrasting sugarcane genotypes. PLoS ONE 2020, 15, e0235845. [CrossRef]

61. Dai, L.; Li, J.; Harmens, H.; Zheng, X.; Zhang, C. Melatonin enhances drought resistance by regulating leaf stomatal behaviour, root growth and catalase activity in two contrasting rapeseed (Brassica napus L.) genotypes. Plant Physiol. Biochem. 2020, 149, 86-95. [CrossRef] [PubMed]

62. Ahmad, P.; Abass Ahanger, M.; Nasser Alyemeni, M.; Wijaya, L.; Alam, P.; Ashraf, M. Mitigation of sodium chloride toxicity in Solanum lycopersicum L. by supplementation of jasmonic acid and nitric oxide. J. Plant Interact. 2018, 13, 64-72. [CrossRef]

63. Blum, A. Osmotic adjustment is a prime drought stress adaptive engine in support of plant production. Plant Cell Environ. 2017, 40, 4-10. [CrossRef] [PubMed]

64. Hayat, S.; Hayat, Q.; Alyemeni, M.N.; Wani, A.S.; Pichtel, J.; Ahmad, A. Role of proline under changing environments. Plant Signal. Behav. 2012, 7, 1456-1466. [CrossRef]

65. Slama, I.; Abdelly, C.; Bouchereau, A.; Flowers, T.; Savouré, A. Diversity, distribution and roles of osmoprotective compounds accumulated in halophytes under abiotic stress. Ann. Bot. 2015, 115, 433-447. [CrossRef]

66. Müller, T.; Lentzsch, P.; Müller, M.E.H. Carbohydrate Dynamics in Leaves of Rapeseed (Brassica napus) Under Drought. J. Agron. Crop Sci. 2012, 198, 207-217. [CrossRef]

67. Cao, Y.; Luo, Q.; Tian, Y.; Meng, F. Physiological and proteomic analyses of the drought stress response in Amygdalus Mira (Koehne) Yü et Lu roots. BMC Plant Biol. 2017, 17, 53. [CrossRef] 